\title{
Mathematical and numerical analysis of a simplified time-dependent viscoelastic flow
}

\author{
Andrea Bonito - Philippe Clément . \\ Marco Picasso
}

Received: 26 September 2006 / Published online: 11 May 2007

(C) Springer-Verlag 2007

\begin{abstract}
A time-dependent model corresponding to an Oldroyd-B viscoelastic fluid is considered, the convective terms being disregarded. Global existence in time is proved in Banach spaces provided the data are small enough, using the implicit function theorem and a maximum regularity property for a three fields Stokes problem. A finite element discretization in space is then proposed. Existence of the numerical solution is proved for small data, so as a priori error estimates, using again an implicit function theorem.
\end{abstract}

Keywords Viscoelastic fluids · Galerkin Least Square finite elements · Semigroup · Perturbed abstract Cauchy problem

Mathematics Subject Classification (2000) 76M10 - 65N15 - 65N30 - 76A10 . 35L05

\section{Introduction}

Numerical modeling of viscoelastic flows is of great importance for complex engineering applications involving foodstuff, blood, paints or adhesives. When considering viscoelastic flows, the velocity, pressure and stress must satisfy the mass and momen-

Supported by the Swiss National Science Foundation. Fellowship PBEL2-114311.

A. Bonito $(\varangle) \cdot$ M. Picasso

Institut d'Analyse et Calcul Scientifique, Ecole Polytechnique Fédérale de Lausanne,

1015 Lausanne, Switzerland

e-mail: andrea.bonito@a3.epfl.ch

P. Clément

Mathematical Institute, Leiden University, P.O. Box 9512, NL-2300 RA, Leiden, The Netherlands 
tum equation, supplemented with a constitutive equation involving the velocity and stress. The simplest model is the so-called Oldroyd-B constitutive relation which can be derived from the kinetic theory of polymer dilute solutions, see for instance $[6,40]$. The unknowns of the Oldroyd-B model are the velocity $u$, the pressure $p$, the extrastress $\sigma$ (the non Newtonian part of the stress due to polymer chains for instance) which must satisfy :

$$
\begin{aligned}
& \rho \frac{\partial u}{\partial t}+\rho(u \cdot \nabla) u-2 \eta_{s} \operatorname{div} \epsilon(u)+\nabla p-\operatorname{div} \sigma=f \\
& \quad \operatorname{div} u=0 \\
& \sigma+\lambda\left(\frac{\partial \sigma}{\partial t}+(u \cdot \nabla) \sigma-(\nabla u) \sigma-\sigma(\nabla u)^{T}\right)-2 \eta_{p} \epsilon(u)=0 .
\end{aligned}
$$

Here $\rho$ is the density, $f$ a force term, $\eta_{s}$ and $\eta_{p}$ are the solvent and polymer viscosities, $\lambda$ the relaxation time, $\epsilon(u)=\frac{1}{2}\left(\nabla u+\nabla u^{T}\right)$ the strain rate tensor, $(\nabla u) \sigma$ denotes the matrix-matrix product between $\nabla u$ and $\sigma$.

Obviously, when $\lambda=0$, the Oldroyd-B model reduces to the incompressible Navier-Stokes equations, for which we refer to [33].

Although the Oldroyd-B model is too simple to describe complex experiments such as shear thinning for instance, it already contains some mathematical and numerical difficulties. Indeed, when solving numerically Oldroyd-B fluids, one is faced to the "high Deborah (or Weissenberg) number problem", that is to say a breakdown in convergence of algorithms when the Deborah number (the relaxation time $\lambda$ times a characteristic velocity divided by a characteristic length) increases. The sources of this problem are due to :

(i) the presence of the quadratic term $(\nabla u) \sigma+\sigma(\nabla u)^{T}$ which prevents a priori estimates to be obtained and therefore existence to be proved for any data;

(ii) the presence of a convective term $(u \cdot \nabla) \sigma$ which requires the use of numerical schemes suited to transport dominated problems;

(iii) the case $\eta_{s}=0$ which requires either a compatibility condition between the finite element spaces for $u$ and $\sigma$ or the use of adequate stabilization procedures such as EVSS for instance.

For a description of numerical procedures used for solving viscoelastic flows in the engineering community, we refer for instance to [3,41].

Concerning mathematical analysis, the existence of slow steady viscoelastic flow has been proved in [45]. For the time-dependent case, existence of solutions locally in time and, for small data, globally in time has been proved in [34] in Hilbert spaces. Extensions to Banach spaces and a review can be found in [25]. Finally, existence for any data has been proved in [36] for a corotational Oldroyd model only.

From the numerical analysis viewpoint, convergence of finite element methods for the linear three fields Stokes problem have been studied for instance in $[11,26,27,46]$. Convergence of continuous and discontinuous finite element methods for steady state viscoelastic fluids have been presented in $[4,24,39,47]$, provided the solution of the continuous problem is smooth and small enough. Extension to time-dependent problems have been proposed in $[5,22,23,38]$. 
In this paper, the mathematical and numerical analysis is proposed for a simplified time-dependent Oldroyd-B problem. More precisely, we focus on item i) above, thus remove the convective terms and assume $\eta_{s}>0$. The reason for considering the time-dependent Oldroyd-B problem without convection is motivated by the fact that this simplified problem corresponds to the correction step in the splitting algorithm described in [10] for solving 3D viscoelastic flows with complex free surfaces. The consequence when removing convective terms is that the implicit function theorem can be used to prove an existence result, whenever the data are small enough, in accordance with the results of [25]. Our existence result is obtained using the semi-group framework and a maximum regularity property for the three fields Stokes problem. Moreover, the regularity of the solution is sufficient to prove convergence of a finite element discretization in space. Finally, it should be noted that the analysis remains valid for more realistic fluids such as Giesekus or Phan-Thien-Tanner.

Throughout the paper, implicit function theorems are used to prove mathematical existence and also numerical convergence of the finite element method, thus the techniques presented in [43] are extended to the time-dependent framework. Finally, we would like to mention that the technics presented here has also been successfully extended to the simplest kinetic model, namely the so-called Hookean dumbbells model for dilute polymer liquids [7-9]. In the latter, the constitutive equation is replaced by a stochastic differential equation for the dumbbells elongation. We refer to [35] for a review concerning numerical methods for kinetic theories of liquid polymers.

The outline of the paper is as follows. The simplified Oldroyd-B problem and its finite element approximation in space are introduced in the next section. Then, in Sect. 3, mathematical existence of a solution is proved in Banach spaces. Finally, existence and a priori error estimates are proposed for the finite element approximation in Sect. 4.

\section{The simplified Oldroyd-B problem and its finite element approximation in space}

Let $\Omega$ be a bounded, connected open set of $\mathbb{R}^{d}, d \geq 2$ with boundary $\partial \Omega$ of class $\mathcal{C}^{2}$, and let $T>0$. We consider the following problem. Given initial conditions $u_{0}: \Omega \rightarrow$ $\mathbb{R}^{d}, \sigma_{0}: \Omega \rightarrow \mathbb{R}_{s y m}^{d \times d}$, a force term $f$, constant solvent and polymer viscosities $\eta_{s}>0$, $\eta_{p}>0$, a constant relaxation time $\lambda>0$, find the velocity $u: \Omega \times(0, T) \rightarrow \mathbb{R}^{d}$, pressure $p: \Omega \times(0, T) \rightarrow \mathbb{R}$ and extra-stress $\sigma: \Omega \times(0, T) \rightarrow \mathbb{R}_{\text {sym }}^{d \times d}$ such that

$$
\begin{aligned}
& \rho \frac{\partial u}{\partial t}-2 \eta_{s} \operatorname{div} \epsilon(u)+\nabla p-\operatorname{div} \sigma=f \text { in } \Omega \times(0, T), \\
& \operatorname{div} u=0 \text { in } \Omega \times(0, T), \\
& \frac{1}{2 \eta_{p}} \sigma+\frac{\lambda}{2 \eta_{p}}\left(\frac{\partial \sigma}{\partial t}-(\nabla u) \sigma-\sigma(\nabla u)^{T}\right) \\
& -\epsilon(u)=0 \text { in } \Omega \times(0, T), \\
& u(\cdot, 0)=u_{0} \text { in } \Omega
\end{aligned}
$$




$$
\begin{aligned}
& \sigma(\cdot, 0)=\sigma_{0} \text { in } \Omega, \\
& u=0 \text { on } \partial \Omega \times(0, T) .
\end{aligned}
$$

For simplicity, the notation will be abridged as follow whenever there is no possible confusion. For $1<r<+\infty$, the space $L^{r}$ denotes $L^{r}(\Omega ; \mathbb{R}), L^{r}\left(\Omega ; \mathbb{R}^{d}\right)$ or $L^{r}\left(\Omega ; \mathbb{R}_{\text {sym }}^{d \times d}\right)$. Also, for $1<q<+\infty, 1<r<+\infty, L^{q}\left(L^{r}\right)$ stands for $L^{q}\left(0, T ; L^{r}(\Omega ; \mathbb{R})\right), L^{q}\left(0, T ; L^{r}\left(\Omega ; \mathbb{R}^{d}\right)\right)$ or $L^{q}\left(0, T ; L^{r}\left(D ; \mathbb{R}_{s y m}^{d \times d}\right)\right)$ and, for $0<$ $\mu<1,1<r<+\infty, \mathcal{C}^{\mu}\left(L^{r}\right)$ stands for the classical Hölder space $\mathcal{C}^{\mu}\left([0, T] ; L^{r}\right.$ $(D ; \mathbb{R}))$. Similarly, $h^{\mu}\left(L^{r}\right)$ stands for the little Hölder space $h^{\mu}\left([0, T] ; L^{r}(D ; \mathbb{R})\right)$. Here, the little Hölder space $h^{\mu}([0, T] ; B)$ is defined for all Banach space $B$ and for all $0<\mu<1$ by

$$
\begin{aligned}
h^{\mu}([0, T] ; B):= & \left\{f \in \mathcal{C}^{\mu}([0, T] ; B) ;\right. \\
& \left.\lim _{\delta \rightarrow 0} \sup _{t, s \in[0, T],|t-s|<\delta} \frac{\|f(t)-f(s)\|_{B}}{|t-s|^{\mu}}=0\right\} .
\end{aligned}
$$

Assuming $B$ is a separable Banach space, the space $h^{k}([0, T] ; B)$ provided with the norm of $\mathcal{C}^{\mu}(0, T ; B)$ is a separable Banach space and for all $0<\mu<\mu^{\prime}<1$ we have $\mathcal{C}^{\mu^{\prime}} \subset h^{\mu}$, see for instance [37]. We also denote by $h_{0}^{\mu}([0, T] ; B)$ the restriction of functions of $h^{\mu}([0, T] ; B)$ vanishing at the origin. The above notations apply for higher order spaces such as $W^{1, q}\left(W^{1, r}\right)$ and $h^{1+\mu}\left(W^{1, r}\right)$.

The implicit function theorem will be used to prove that (1)-(6) admits a unique solution

$$
\begin{aligned}
& u \in W^{1, q}\left(L^{r}\right) \cap L^{q}\left(W^{2, r} \cap H_{0}^{1}\right), \quad p \in L^{q}\left(W^{1, r} \cap L_{0}^{2}\right), \\
& \sigma \in W^{1, q}\left(W^{1, r}\right),
\end{aligned}
$$

or

$$
\begin{aligned}
& u \in h^{1+\mu}\left(L^{r}\right) \cap h^{\mu}\left(W^{2, r} \cap H_{0}^{1}\right), \quad p \in h^{\mu}\left(W^{1, r} \cap L_{0}^{2}\right), \\
& \sigma \in h^{1+\mu}\left(W^{1, r}\right),
\end{aligned}
$$

with $1<q<\infty, d<r<\infty$ and $0<\mu<1$ for any data $f, u_{0}, \sigma_{0}$ small enough in appropriate spaces. Moreover, assuming more regularity on the data, we will also prove that

$$
\begin{aligned}
& u \in W^{2, q}\left(L^{r}\right) \cap W^{1, q}\left(W^{2, r} \cap H_{0}^{1}\right), \quad p \in W^{1, q}\left(W^{1, r} \cap L_{0}^{2}\right), \\
& \sigma \in W^{2, q}\left(W^{1, r}\right),
\end{aligned}
$$

or

$$
\begin{aligned}
& u \in h^{2+\mu}\left(L^{r}\right) \cap h^{1+\mu}\left(W^{2, r} \cap H_{0}^{1}\right), \quad p \in h^{1+\mu}\left(W^{1, r} \cap L_{0}^{2}\right), \\
& \sigma \in h^{2+\mu}\left(W^{1, r}\right),
\end{aligned}
$$


for any data $f, u_{0}, \sigma_{0}$ again small enough in appropriate spaces. The regularity (7) is sufficient to prove convergence of a finite element discretization in space, see Sect. 4. On the other hand, the regularity (9) will be needed to prove convergence of a space and time discretization, this being the subject of a forthcoming paper. Finally, the regularities (8) and (10) will be used when considering the Hookean dumbbells model, which is formally equivalent to the Oldroyd-B model, see for instance [40]. Analysis and numerical analysis of this stochastic model will be considered elsewhere [7].

Alternatively, local existence in time is proved for arbitrarily large data, using an abstract theorem for fully nonlinear parabolic equations, namely Theorem 8.1.1 of [37]. More precisely, we will prove that there exists $0<T_{*} \leq T$ such that (1)-(6) admits a solution

$$
\begin{aligned}
& u \in \mathcal{C}^{1}\left(\left[0, T_{*}\right], L^{r}\right) \cap \mathcal{C}^{0}\left(\left[0, T_{*}\right], W^{2, r} \cap H_{0}^{1}\right), \\
& \sigma \in \mathcal{C}^{1}\left(\left[0, T_{*}\right], W^{1, r}\right), \\
& p \in \mathcal{C}^{0}\left(\left[0, T_{*}\right] ; W^{1, r} \cap L_{0}^{2}\right),
\end{aligned}
$$

with $d<r<\infty$ and for any data $f, u_{0}$ and $\sigma_{0}$ in appropriate spaces.

The finite element approximation in space is now introduced. For any $h>0$, let $\mathcal{T}_{h}$ be a decomposition of $\Omega$ into triangles $K$ with diameter $h_{K}$ less than $h$, regular in the sense of [17]. We consider $V_{h}, M_{h}$ and $Q_{h}$ the finite element spaces for the velocity, extra-stress and pressure, respectively defined by :

$$
\begin{aligned}
V_{h} & :=\left\{v_{h} \in \mathcal{C}^{0}\left(\bar{\Omega} ; \mathbb{R}^{d}\right) ;\left.v_{h}\right|_{K} \in\left(\mathbb{P}_{1}\right)^{d}, \forall K \in \mathcal{T}_{h}\right\} \cap H_{0}^{1}\left(\Omega ; \mathbb{R}^{d}\right), \\
M_{h} & :=\left\{\tau_{h} \in \mathcal{C}^{0}\left(\bar{\Omega} ; \mathbb{R}_{\text {sym }}^{d \times d}\right) ;\left.\tau_{h}\right|_{K} \in\left(\mathbb{P}_{1}\right)_{\text {sym }}^{d \times d}, \forall K \in \mathcal{T}_{h}\right\}, \\
Q_{h} & :=\left\{q_{h} \in \mathcal{C}^{0}(\bar{\Omega} ; \mathbb{R}) ;\left.q_{h}\right|_{K} \in \mathbb{P}_{1}, \forall K \in \mathcal{T}_{h}\right\} \cap L_{0}^{2}(\Omega ; \mathbb{R}) .
\end{aligned}
$$

We denote $i_{h}$ the $L^{2}(\Omega)$ projection onto $V_{h}, M_{h}$ or $Q_{h}$ and introduce the following stabilized finite element discretization in space of (1)-(6). Given $f, u_{0}, \sigma_{0}$ find

$$
\left(u_{h}, \sigma_{h}, p_{h}\right): t \rightarrow\left(u_{h}(t), \sigma_{h}(t), p_{h}(t)\right) \in V_{h} \times M_{h} \times Q_{h}
$$

such that $u_{h}(0)=i_{h} u_{0}, \sigma_{h}(0)=i_{h} \sigma_{0}$ and such that the following weak formulation holds in ]0, $T$ [ :

$$
\begin{aligned}
& \rho\left(\frac{\partial u_{h}}{\partial t}, v_{h}\right)+2 \eta_{s}\left(\epsilon\left(u_{h}\right), \epsilon\left(v_{h}\right)\right)-\left(p_{h}, \operatorname{div} v_{h}\right)+\left(\sigma_{h}, \epsilon\left(v_{h}\right)\right) \\
& -\left(f, v_{h}\right)+\left(\operatorname{div} u_{h}, q_{h}\right)+\sum_{K \in \mathcal{T}_{h}} \frac{\alpha h_{K}^{2}}{2 \eta_{p}}\left(\nabla p_{h}, \nabla q_{h}\right)_{K} \\
& \quad+\frac{1}{2 \eta_{p}}\left(\sigma_{h}, \tau_{h}\right)+\frac{\lambda}{2 \eta_{p}}\left(\frac{\partial \sigma_{h}}{\partial t}-\left(\nabla u_{h}\right) \sigma_{h}-\sigma_{h}\left(\nabla u_{h}\right)^{T}, \tau_{h}\right) \\
& -\left(\epsilon\left(u_{h}\right), \tau_{h}\right)=0
\end{aligned}
$$


for all $\left(v_{h}, \tau_{h}, q_{h}\right) \in V_{h} \times M_{h} \times Q_{h}$. Here $\alpha>0$ is a dimensionless stabilization parameter and $(\cdot, \cdot)$ (respectively $(\cdot, \cdot)_{K}$ ) denotes the $L^{2}(\Omega)$ (resp. $L^{2}(K)$ ) scalar product for scalars, vectors and tensors.

The above nonlinear finite element scheme has already being studied in the stationary case [43]. Indeed, using the convergence result of [11] for the linear three fields Stokes problem and an implicit function theorem taken from [12,13,15], existence and convergence could be proved for small $\lambda$, the difficulty being again due to the fact that no a priori estimates can be obtained because of the presence of the quadratic terms $\left(\nabla u_{h}\right) \sigma_{h}+\sigma_{h}\left(\nabla u_{h}\right)^{T}$.

We will proceed in an analogous manner for the time dependent case and prove existence and convergence of a solution to (12) for a given $\lambda$ but for small data $f, u_{0}$, $\sigma_{0}$. It should be noted that in this paper the case $\eta_{s}=0$ is not considered, therefore some of the stabilization terms present in $[11,43]$ are not included in the finite element formulation (12).

\section{Existence of a solution to the simplified Oldroyd-B problem}

We introduce, as in [25], the Helmholtz-Weyl projector [28-30] defined by

$$
P_{r}: L^{r}\left(\Omega ; \mathbb{R}^{d}\right) \rightarrow \mathcal{H}_{r} \quad 1<r<\infty
$$

where $\mathcal{H}_{r}$ is the completion of the divergence free $C_{0}^{\infty}(\Omega)$ vector fields with respect to the $L^{r}$ norm. The space $\mathcal{H}_{r}$ can be characterized as follows (again see for instance [29])

$$
\mathcal{H}_{r}=\left\{v \in L^{r}\left(\Omega ; \mathbb{R}^{d}\right) ; \operatorname{div} v=0, v \cdot n=0 \text { on } \partial \Omega\right\}
$$

Since $\Omega$ is of class $\mathcal{C}^{2}$, there exists a constant $C$ such that for all $f \in L^{r}$

$$
\left\|P_{r} f\right\|_{L^{r}} \leq C\|f\|_{L^{r}}
$$

We define $A_{r}:=-P_{r} \Delta: \mathcal{D}_{A_{r}} \rightarrow \mathcal{H}_{r}$ the Stokes operator, where

$$
\mathcal{D}_{A_{r}}:=\left\{v \in W^{2, r}\left(\Omega ; \mathbb{R}^{d}\right) \cap W_{0}^{1, r}\left(\Omega ; \mathbb{R}^{d}\right) \mid \operatorname{div} v=0\right\}
$$

It is well known (see [32] for instance) that, for $\Omega$ of class $\mathcal{C}^{2}$, the operator $A_{r}$ equipped with the usual norm of $L^{r}\left(\Omega ; \mathbb{R}^{d}\right)$ is closed and densely defined in $\mathcal{H}_{r}$. Moreover, the graph norm of $A_{r}$ is equivalent to the $W^{2, r}$ norm.

With the above operators, $(u, \sigma)$ is said to be a solution of (1)-(6) if

$$
u \in W^{1, q}\left(\mathcal{H}_{r}\right) \cap L^{q}\left(\mathcal{D}_{A_{r}}\right), \quad \sigma \in W^{1, q}\left(W^{1, r}\right),
$$


with $1<q<\infty, d<r<\infty$ and satisfies

$$
\begin{aligned}
& \rho \frac{\partial u}{\partial t}+\eta_{s} A_{r} u-P_{r} \operatorname{div} \sigma=P_{r} f \\
& \frac{1}{2 \eta_{p}} \sigma+\frac{\lambda}{2 \eta_{p}}\left(\frac{\partial \sigma}{\partial t}-(\nabla u) \sigma-\sigma(\nabla u)^{T}\right)-\epsilon(u)=0 \\
& u(\cdot, 0)=u_{0} \\
& \sigma(\cdot, 0)=\sigma_{0} .
\end{aligned}
$$

We will assume that the source term is $f \in L^{q}\left(L^{r}\right)$, the initial data are $u_{0} \in E_{1-1 / q, q}$ and $\sigma_{0} \in W^{1, r}$. Here $E_{1-1 / q, q}:=\left(\mathcal{H}_{r}, \mathcal{D}_{A_{r}}\right)_{1-1 / q, q}$ is a real interpolation space which can be defined as

$$
E_{1-1 / q, q}:=\left\{v \in \mathcal{H}_{r} ; \int_{0}^{+\infty}\left\|A_{r} e^{-t A_{r}} v\right\|_{L_{r}}^{q}<\infty\right\}
$$

and is a Banach space with norm

$$
\|v\|_{E_{1-1 / q, q}}:=\|v\|_{L_{r}}+\left(\int_{0}^{+\infty}\left\|A_{r} e^{-t A_{r}} v\right\|_{L_{r}}^{q}\right)^{1 / q} .
$$

Moreover, when considering the little Hölder spaces $h^{\mu}\left(L^{r}\right)$ - see the regularities (8) and (10) - the space $E_{\mu, \infty}:=\left(\mathcal{H}_{r}, \mathcal{D}_{A_{r}}\right)_{\mu, \infty}$ will also be needed. The space $E_{\mu, \infty}$ can be defined as

$$
E_{\mu, \infty}=\left\{v \in \mathcal{H}_{r} ; \sup _{t>0}\left\|t^{1-\mu} A_{r} e^{-t A_{r}} v\right\|_{L_{r}}<+\infty\right\}
$$

and is a Banach space endowed with the norm

$$
\|v\|_{E_{\mu, \infty}}:=\|v\|_{L_{r}}+\sup _{t>0}\left\|t^{1-\mu} A_{r} e^{-t A_{r}} v\right\|_{L_{r}} .
$$

We refer to $[18,19,49]$ for more details.

Remark 1 For $1<q<+\infty, d<r<+\infty$, we have

$$
W^{1, q}\left(\mathcal{H}_{r}\right) \cap L^{q}\left(\mathcal{D}_{A_{r}}\right) \subset L^{2}\left(W_{0}^{1, r}\right),
$$

(see [21,32,51]). Thus a solution of (13)-(16) satisfies

$$
\|u(T)\|_{L^{2}}^{2}+\|\nabla u\|_{L^{2}\left(L^{2}\right)}^{2}<\infty .
$$


Uniqueness of a solution to problem (13)-(16) can be obtained proceeding as in [25], that is to say by proving an a priori estimate for the difference of two solutions when $q \geq 2$.

Lemma 1 Let $d \geq 2$, let $\Omega \subset \mathbb{R}^{d}$ be a bounded, connected open set with boundary of class $\mathcal{C}^{2}$, let $T>0$ and assume $2 \leq q<\infty, d<r<\infty$. Then, for any $f \in L^{q}\left(L^{r}\right), u_{0} \in E_{1-1 / q, q}, \sigma_{0} \in W^{1, r}$, there exists at most one solution $(u, \sigma)$ of problem (13)-(16).

Proof Let us start by noticing that for $1 \leq q<\infty, d<r<\infty, v \in L^{q}\left(W^{2, r}\right)$ and $\tau \in W^{1, q}\left(W^{1, r}\right)$ the nonlinearity $(\nabla v) \tau+\tau(\nabla v)^{T} \in L^{q}\left(W^{1, r}\right)$. Indeed, $W^{1, r}$ is an algebra for $d<r$ and there exists $C>0$ independent of $v$ and $\tau$ such that

$$
\left\|(\nabla v) \tau+\tau(\nabla v)^{T}\right\|_{W^{1, r}} \leq C\|\tau\|_{W^{1, r}}\|v\|_{W^{1, r}},
$$

see for instance Theorem 5.23 of [1]. Moreover, using the same arguments as in [1], we have that $W^{1, q}(B) \subset_{>} L^{\infty}(B)$ for all Banach space $B$, thus

$$
\begin{aligned}
\|(\nabla v) \tau\|_{L^{q}\left(W^{1, r}\right)}^{q} & =\int_{0}^{T}\|(\nabla v) \tau\|_{W^{1, r}}^{q} \leq C \int_{0}^{T}\|\nabla v\|_{W^{1, r}}^{q}\|\tau\|_{W^{1, r}}^{q} \\
& \leq C\|\tau\|_{L^{\infty}\left(W^{1, r}\right)}^{q} \int_{0}^{T}\|\nabla v\|_{W^{1, r}}^{q} \\
& \leq \tilde{C}\|\tau\|_{W^{1, q}\left(W^{1, r}\right)}^{q}\|v\|_{L^{q}\left(W^{2, r}\right)}^{q},
\end{aligned}
$$

where $\tilde{C}$ is independent of $v$ and $\tau$. Therefore, we can define the mapping $S$ : $L^{q}\left(W^{2, r}\right) \times W^{1, q}\left(W^{1, r}\right) \rightarrow L^{q}\left(W^{1, r}\right)$ by

$$
S(v, \tau):=\frac{\lambda}{2 \eta_{p}}\left((\nabla v) \tau+\tau(\nabla v)^{T}\right) .
$$

Let now $\left(u_{i}, \sigma_{i}\right) \in W^{1, q}\left(\mathcal{H}_{r}\right) \cap L^{q}\left(\mathcal{D}_{A_{r}}\right) \times W^{1, q}\left(W^{1, r}\right) i=1$, 2, be two solutions of problem (13)-(16) and let $u=u_{1}-u_{2}, \sigma=\sigma_{1}-\sigma_{2}$. Using the well known properties of the Helmholtz-Weyl projector [28-30], there exists a unique pressure $p_{i} \in L^{q}\left(W^{1, r} \cap L_{0}^{2}\right), i=1,2$, corresponding to each pair $\left(u_{i}, \sigma_{i}\right)$ such that $\left(u_{i}, \sigma_{i}, p_{i}\right)$ satisfies (1)-(6). When $2 \leq q<+\infty$, we can then take the weak formulation to obtain

$$
\begin{aligned}
& \rho \int_{0}^{t}\left(\frac{\partial u_{i}}{\partial t}, u\right)+\frac{\lambda}{2 \eta_{p}} \int_{0}^{t}\left(\frac{\partial \sigma_{i}}{\partial t}, \sigma\right)+\eta_{s} \int_{0}^{t}\left(\nabla u_{i}, \nabla u\right) \\
& +\int_{0}^{t}\left(\sigma_{i}, \epsilon(u)\right)+\frac{1}{2 \eta_{p}} \int_{0}^{t}\left(\sigma_{i}, \sigma\right)-\int_{0}^{t}\left(\epsilon\left(u_{i}\right), \sigma\right)=\int_{0}^{t}\left(S\left(u_{i}, \sigma_{i}\right), \sigma\right),
\end{aligned}
$$


for $i=1,2$. Hereabove we have used the fact that, since div $u_{i}=0$, we have

$$
2 \operatorname{div} \epsilon\left(u_{i}\right)=\Delta u_{i}
$$

All the terms in the previous equation are well defined because of the regularity of $u_{i}$ and $\sigma_{i}$ and since $u(0)=\sigma(0)=0$ we have

$$
\rho \int_{0}^{t}\left(\frac{\partial u_{i}}{\partial t}, u\right)+\frac{\lambda}{2 \eta_{p}} \int_{0}^{t}\left(\frac{\partial \sigma_{i}}{\partial t}, \sigma\right)=\frac{\rho}{2}\|u(t)\|_{L^{2}}^{2}+\frac{\lambda}{4 \eta_{p}}\|\sigma(t)\|_{L^{2}}^{2},
$$

for $i=1,2$ and $t \in(0, T)$. Subtracting the two equalities (20), it follows that

$$
\begin{aligned}
& \left(\frac{\rho}{2}\|u(t)\|_{L^{2}}^{2}+\frac{\lambda}{4 \eta_{p}}\|\sigma(t)\|_{L^{2}}^{2}\right)+\eta_{s}\|\nabla u\|_{L^{2}\left(L^{2}\right)}^{2}+\frac{1}{2 \eta_{p}}\|\sigma\|_{L^{2}\left(L^{2}\right)}^{2} \\
& \quad=\int_{0}^{t}\left(S\left(u, \sigma_{1}\right), \sigma\right)+\int_{0}^{t}\left(S\left(u_{2}, \sigma\right), \sigma\right) .
\end{aligned}
$$

Then, using Cauchy-Schwarz and Young inequalities, we have for $t \in(0, T)$

$$
\begin{aligned}
\int_{0}^{t}\left(S\left(u, \sigma_{1}\right), \sigma\right) & \leq \frac{2 \lambda}{2 \eta_{p}} \int_{0}^{t}\left\|\sigma_{1}\right\|_{L^{\infty}}\|\nabla u\|_{L^{2}}\|\sigma\|_{L^{2}} \\
& \leq \frac{\lambda^{2}}{2 \eta_{s} \eta_{p}^{2}} \int_{0}^{t}\left\|\sigma_{1}\right\|_{L^{\infty}}^{2}\|\sigma\|_{L^{2}}^{2}+\frac{\eta_{s}}{2} \int_{0}^{t}\|\nabla u\|_{L^{2}}^{2}
\end{aligned}
$$

and

$$
\int_{0}^{t}\left(S\left(u_{2}, \sigma\right), \sigma\right) \leq \frac{\lambda}{2 \eta_{p}} \int_{0}^{t}\left\|\nabla u_{2}\right\|_{L^{\infty}}\|\sigma\|_{L^{2}}^{2} .
$$

Hence, with (21) and the continuous injection $W^{1, r} \subset_{>} L^{\infty}$ it follows that

$$
\frac{\rho}{2}\|u(t)\|_{L^{2}}^{2}+\frac{\lambda}{4 \eta_{p}}\|\sigma(t)\|_{L^{2}}^{2} \leq C \int_{0}^{t}\left(\left\|u_{2}\right\|_{W^{2, r}}+\left\|\sigma_{1}\right\|_{W^{1, r}}^{2}\right)\|\sigma\|_{L^{2}}^{2}
$$

for $t \in(0, T)$. Here $C$ is a constant independent of $u_{1}, u_{2}, \sigma_{1}$ and $\sigma_{2}$. Gronwall's Lemma is used to obtain for all $t \in(0, T)$

$$
\rho\|u(t)\|_{L^{2}}^{2}+\frac{\lambda}{2 \eta_{p}}\|\sigma(t)\|_{L^{2}}^{2}=0
$$

so that $u \in W^{1, q}\left(\mathcal{H}_{r}\right) \cap L^{q}\left(\mathcal{D}_{A_{r}}\right)$ and $\sigma \in W^{1, q}\left(W^{1, r}\right)$ vanish.

We can now state the main results of this section. 
Theorem 1 Let $d \geq 2$, let $\Omega \subset \mathbb{R}^{d}$ be a bounded, connected open set with boundary of class $\mathcal{C}^{2}$, let $T>0$ and assume $d<r<\infty, 1<q<\infty, 0<\mu<1$. Then, there exists $\delta_{0}>0$ such that the following holds.

(i) If $f \in L^{q}\left(L^{r}\right), u_{0} \in E_{1-1 / q, q}, \sigma_{0} \in W^{1, r}$ satisfy

$$
\left\|P_{r} f\right\|_{L^{q}\left(L^{r}\right)}+\left\|u_{0}\right\|_{E_{1-1 / q, q}}+\left\|\sigma_{0}\right\|_{W^{1, r}} \leq \delta_{0},
$$

then there exists a solution of (13)-(16).

(ii) If $f \in W^{1, q}\left(L^{r}\right), u_{0} \in \mathcal{D}_{A_{r}}, \sigma_{0} \in W^{1, r}$ satisfy the compatibility condition

$$
-\eta_{s} A_{r} u_{0}+P_{r} f(0)+P_{r} \operatorname{div} \sigma_{0} \in E_{1-1 / q, q}
$$

and are such that

$$
\begin{aligned}
& \left\|P_{r} f\right\|_{W^{1, q}\left(L^{r}\right)}+\left\|u_{0}\right\|_{W^{2, r}}+\left\|\sigma_{0}\right\|_{W^{1, r}} \\
& \quad+\left\|-\eta_{s} A_{r} u_{0}+P_{r} f(0)+P_{r} \operatorname{div} \sigma_{0}\right\|_{E_{1-1 / q, q}} \leq \delta_{0},
\end{aligned}
$$

then there exists a solution of (13)-(16) with

$$
u \in W^{2, q}\left(\mathcal{H}_{r}\right) \cap W^{1, q}\left(\mathcal{D}_{A_{r}}\right), \quad \sigma \in W^{2, q}\left(W^{1, r}\right) .
$$

(iii) If $f \in h^{\mu}\left(L^{r}\right), u_{0} \in \mathcal{D}_{A_{r}}, \sigma_{0} \in W^{1, r}$ satisfying the compatibility condition

$$
-\eta_{s} A_{r} u_{0}+P_{r} f(0)+P_{r} \operatorname{div} \sigma_{0} \in \overline{\mathcal{D}}_{A_{r}} E_{\mu, \infty}
$$

and are such that

$$
\begin{aligned}
& \left\|P_{r}(f-f(0))\right\|_{h^{\mu}\left(L^{r}\right)}+\left\|u_{0}\right\|_{W^{2, r}}+\left\|\sigma_{0}\right\|_{W^{1, r}} \\
& \quad+\left\|-\eta_{s} A_{r} u_{0}+P_{r} f(0)+P_{r} \operatorname{div} \sigma_{0}\right\|_{\overline{\mathcal{D}}_{A_{r}}} E_{\mu, \infty} \leq \delta_{0},
\end{aligned}
$$

then there exists a solution of (13)-(16) with

$$
u \in h^{1+\mu}\left(\mathcal{H}_{r}\right) \cap h^{\mu}\left(\mathcal{D}_{A_{r}}\right), \quad \sigma \in h^{1+\mu}\left(W^{1, r}\right) .
$$

(iv) If $f \in h^{1+\mu}\left(L^{r}\right), u_{0} \in \mathcal{D}_{A_{r}}, \sigma_{0} \in W^{1, r}$ satisfying the compatibility conditions

$$
-\eta_{s} A_{r} u_{0}+P_{r} f(0)+P_{r} \operatorname{div} \sigma_{0} \in \mathcal{D}\left(A_{r}\right),
$$

$$
-\eta_{s} A_{r}\left(-\eta_{s} A_{r} u_{0}+P_{r} f(0)+P_{r} \operatorname{div} \sigma_{0}\right)+\frac{\partial f}{\partial t}(0) \in \overline{\mathcal{D}}_{A_{r}} E_{\mu, \infty},
$$


and are such that

$$
\begin{aligned}
& \left\|P_{r}(f-f(0))\right\|_{h^{\mu}\left(L^{r}\right)}+\left\|P_{r}\left(\frac{\partial f}{\partial t}-\frac{\partial f}{\partial t}(0)\right)\right\|_{h^{\mu}\left(L^{r}\right)}+\left\|u_{0}\right\|_{W^{2, r}}+\left\|\sigma_{0}\right\|_{W^{1, r}} \\
& \quad+\left\|-\eta_{s} A_{r} u_{0}+P_{r} f(0)+P_{r} \operatorname{div} \sigma_{0}\right\|_{W^{2, r}} \\
& \quad+\left\|-\eta_{s} A_{r}\left(-\eta_{s} A_{r} u_{0}+P_{r} f(0)+P_{r} \operatorname{div} \sigma_{0}\right)+\frac{\partial f}{\partial t}(0)\right\|_{\overline{\mathcal{D}}_{A_{r}}} E_{\mu, \infty} \leq \delta_{0},
\end{aligned}
$$

then there exists a solution of (13)-(16) with

$$
u \in h^{2+\mu}\left(\mathcal{H}_{r}\right) \cap h^{1+\mu}\left(\mathcal{D}_{A_{r}}\right), \quad \sigma \in h^{2+\mu}\left(W^{1, r}\right) .
$$

Moreover, in all cases, the mappings

$$
\left(P_{r} f, u_{0}, \sigma_{0}\right) \mapsto\left(u\left(P_{r} f, u_{0}, \sigma_{0}\right), \sigma\left(P_{r} f, u_{0}, \sigma_{0}\right)\right)
$$

are analytic in their respective spaces.

Using the well known properties of the Helmholtz-Weyl projector [28-30], we can then obtain the following result.

Corollary 1 Under the assumptions of the above Theorem, there exists a unique $p$ satisfying

$$
\begin{aligned}
& \text { (i) } p \in L^{q}\left(W^{1, r} \cap L_{0}^{2}\right), \\
& \text { (ii) } p \in W^{1, q}\left(W^{1, r} \cap L_{0}^{2}\right), \\
& \text { (iii) } p \in h^{\mu}\left(W^{1, r} \cap L_{0}^{2}\right), \\
& \text { (iv) } p \in h^{1+\mu}\left(W^{1, r} \cap L_{0}^{2}\right),
\end{aligned}
$$

such that $(u, \sigma, p)$ is solution of problem (1)-(6). Moreover, the mappings

$$
\left(f, u_{0}, \sigma_{0}\right) \mapsto\left(u\left(f, u_{0}, \sigma_{0}\right), \sigma\left(f, u_{0}, \sigma_{0}\right), p\left(f, u_{0}, \sigma_{0}\right)\right)
$$

are analytic in their respective spaces.

Local existence in time can be proved for arbitrarily large data, using an abstract theorem for fully nonlinear parabolic equations, namely Theorem 8.1.1 of [37].

Theorem 2 Let $d \geq 2$, let $\Omega \subset \mathbb{R}^{d}$ be a bounded, connected open set with boundary of class $\mathcal{C}^{2}$ and assume $d<r<\infty, 0<\mu<1, T>0$. If

$$
f \in \mathcal{C}^{\mu}\left(L^{r}\right), \quad u_{0} \in \mathcal{D}_{A_{r}}, \quad \sigma_{0} \in W^{1, r},
$$

then there exists $T_{*} \in(0, T]$ such that problem (13)-(16) possesses a solution

$$
u \in \mathcal{C}^{1}\left(\left[0, T_{*}\right], \mathcal{H}_{r}\right) \cap \mathcal{C}^{0}\left(\left[0, T_{*}\right], \mathcal{D}_{A_{r}}\right), \quad \sigma \in \mathcal{C}^{1}\left(\left[0, T_{*}\right], W^{1, r}\right) .
$$


As for Corollary 1 we can deduce the following result.

Corollary 2 Under the assumptions of the above Theorem, there exists a unique

$$
p \in \mathcal{C}^{1}\left(\left[0, T_{*}\right], W^{1, r} \cap L_{0}^{2}\right)
$$

such that $(u, \sigma, p)$ is a solution of problem (1)-(6).

Remark 2 Part (i) of Theorem 1 is compatible with Theorem 9.2 of [25], in which the convective terms have been taken into account. Moreover, if $2 / q+d / r<1$, we have $L^{q}\left(\mathcal{D}_{A_{r}}\right) \cap W^{1, q}\left(\mathcal{H}_{r}\right) \subset_{>} \mathcal{C}^{0}\left(\mathcal{C}^{1}\right)$ and thus $\nabla u \in \mathcal{C}^{0}([0, T] \times \bar{\Omega})$ which implies $(u \cdot \nabla) u \in L^{q}\left(L^{r}\right)$. Therefore, Theorem 1 part (i) still holds when the convective term $(u \cdot \nabla) u$ is added to the momentum equation (1) or (13). However, since $(u \cdot \nabla) \sigma \notin W^{1, q}\left(W^{1, r}\right)$, the convective term $(u \cdot \nabla) \sigma$ can not be added to (14) in the present analysis.

Remark 3 Since

$$
\|(u \cdot \nabla) u\|_{W^{1, q}\left(L^{r}\right)} \leq C\left(\|u\|_{W^{1, q}\left(\mathcal{D}_{A_{r}}\right)}+\|u\|_{W^{2, q}\left(\mathcal{H}_{r}\right)}\right),
$$

then Theorem 1 part (ii) still holds if the convective term $(u \cdot \nabla) u$ is added to (13). However, since $(u \cdot \nabla) \sigma \notin W^{2, q}\left(W^{1, r}\right)$, the convective term $(u \cdot \nabla) \sigma$ can not be added to (14) in the present analysis.

Remark 4 Parts (iii) and (iv) of Theorem 1 still hold when replacing little Hölder spaces by the classical Hölder spaces. Indeed, the only difference is that the trace space is not $\overline{\mathcal{D}_{A_{r}}} E_{\mu, \infty}$ anymore but $E_{\mu, \infty}$.

Remark 5 The trace spaces $E_{1-1 / q, q}$ or $\overline{\mathcal{D}_{A_{r}}} E_{\mu, \infty}$ are abstract space but they both contain $\mathcal{D}_{A_{r}}$. For instance in part (i), if $u_{0} \in W^{2, r} \cap W_{0}^{1, r}$ then $u_{0} \in E_{1-1 / q, q}$. Also, the condition " $\left\|P_{r} f\right\|_{L^{q}\left(L^{r}\right)}$ small" is satisfied whenever $\|f\|_{L^{q}\left(L^{r}\right)}$ is small.

Remark 6 The existence results presented in this section still hold when considering more realistic constitutive equations for the extra-stress tensor $\sigma$. This is for instance the case of the simplified Giesekus [31] and Phan-Thien Tanner [42] models, respectively defined by

$$
\begin{aligned}
& \sigma+\lambda\left(\frac{\partial \sigma}{\partial t}-(\nabla u) \sigma-\sigma(\nabla u)^{T}\right)+\alpha \frac{\lambda}{\eta_{p}} \sigma \sigma=2 \eta_{p} \epsilon(u), \\
& \sigma+\lambda\left(\frac{\partial \sigma}{\partial t}-(\nabla u) \sigma-\sigma(\nabla u)^{T}\right)+\epsilon \frac{\lambda}{\eta_{p}} \operatorname{tr}(\sigma) \sigma=2 \eta_{p} \epsilon(u),
\end{aligned}
$$

where $\alpha$ and $\epsilon$ are given positive parameters.

Remark 7 If we assume that the operator $A_{r}$ satisfies the maximal regularity property (see Definition (1) in Appendix A) when $\Omega$ is a convex polygon, then Theorem 1 still holds. We did not find such a result in the literature, therefore we will make this 
assumption and prove convergence of the finite element scheme. It should be noted that the corresponding property is true in stationary case for some $r>2$ depending on the angles of the polygon, see [43].

\subsection{Proof of Theorem 1}

The proof is detailed for part (ii) only, which contains the major mathematical difficulties. Then, we will briefly explain how the same arguments can be used to prove parts (i), (iii) and (iv).

In order to prove part (ii) of Theorem 1, we shall introduce the mapping $F$ : $Y \times X \rightarrow Z$, where

$$
\begin{aligned}
Y= & \left\{\left(P_{r} f, u_{0}, \sigma_{0}\right), \text { such that }\left(f, u_{0}, \sigma_{0}\right) \in W^{1, q}\left(L^{r}\right) \times \mathcal{D}_{A_{r}} \times W^{1, r}\right. \\
& \left.\quad \text { and }-\eta_{s} A_{r} u_{0}+P_{r} f(0)+P_{r} \operatorname{div} \sigma_{0} \in E_{1-1 / q, q}\right\} \\
X= & W^{2, q}\left(\mathcal{H}_{r}\right) \cap W^{1, q}\left(\mathcal{D}_{A_{r}}\right) \times W^{2, q}\left(W^{1, r}\right) \\
Z= & W^{1, q}\left(W^{1, r}\right) \times Y .
\end{aligned}
$$

The mapping $F$ is defined for all $y=\left(P_{r} f, u_{0}, \sigma_{0}\right) \in Y$ and $x=(u, \sigma) \in X$ by

$$
F(y, x)=\left(\begin{array}{c}
\frac{\lambda}{2 \eta_{p}} \frac{\partial \sigma}{\partial t}+\frac{1}{2 \eta_{p}} \sigma-\epsilon(u)-S(u, \sigma) \\
\rho \frac{\partial u}{\partial t}+\eta_{s} A_{r} u-P_{r} \operatorname{div} \sigma-P_{r} f \\
u(., 0)-u_{0} \\
\sigma(., 0)-\sigma_{0}
\end{array}\right)
$$

with

$$
S(u, \sigma)=\frac{\lambda}{2 \eta_{p}}\left((\nabla u) \sigma+\sigma(\nabla u)^{T}\right) .
$$

Then problem (13)-(16) can be reformulated as follows. Given $y \in Y$, find $x \in X$ such that

$$
F(y, x)=0 \quad \text { in } Z .
$$

The aim is to use the implicit function theorem, hence noticing that $F(0,0)=0$, we will prove that

- the spaces $X, Y$ and $Z$ equipped with appropriate norms are Banach spaces,

- $F$ is a well defined, real analytic mapping,

- the Fréchet derivative $D_{x} F(0,0)$ is an isomorphism from $X$ to $Z$.

This will establish existence for part ii) of Theorem 1. Uniqueness follows from Lemma 1 for $2 \leq q<\infty$.

The space $X$ is equipped with the norm $\|\cdot\|_{X}$ defined for $x=(u, \sigma) \in X$ by

$$
\|x\|_{X}=\|u, \sigma\|_{X}=\|u\|_{W^{2, q}\left(L^{r}\right)}+\|u\|_{W^{1, q}\left(W^{2, r}\right)}+\|\sigma\|_{W^{2, q}\left(W^{1, r}\right)} .
$$


Obviously, $\left(X,\|.\|_{X}\right)$ becomes a Banach space. The space $Y$ is equipped with the norm $\|\cdot\|_{Y}$ defined for $y=\left(P_{r} f, u_{0}, \sigma_{0}\right) \in Y$ by

$$
\begin{aligned}
\|y\|_{Y}= & \left\|P_{r} f, u_{0}, \sigma_{0}\right\|_{Y} \\
= & \left\|P_{r} f\right\|_{W^{1, q}\left(L^{r}\right)}+\left\|u_{0}\right\|_{W^{2, r}}+\left\|\sigma_{0}\right\|_{W^{1, r}} \\
& +\left\|-\eta_{s} A_{r} u_{0}+P_{r} f(0)+P_{r} \operatorname{div} \sigma_{0}\right\|_{E_{1-1 / q, q}} .
\end{aligned}
$$

As a consequence of the continuity of the linear mapping

$$
\left(P_{r} f, u_{0}, \sigma_{0}\right) \longmapsto-\eta_{s} A_{r} u_{0}+P_{r} f(0)+P_{r} \operatorname{div} \sigma_{0}
$$

from $W^{1, q}\left(\mathcal{H}_{r}\right) \times \mathcal{D}_{A_{r}} \times W^{1, r}$ (equipped with the product norm) to $L^{r}$ and due to the completeness of $E_{1-1 / q, q}$, the space $\left(Y,\|.\|_{Y}\right)$ is a closed subspace of $W^{1, q}\left(\mathcal{H}_{r}\right) \times$ $\mathcal{D}_{A_{r}} \times W^{1, r}$ and thus a Banach space. The space $Z$ is equipped with the product norm and becomes a Banach space.

In order to prove that $F$ is well defined and analytic we need to prove that $S: X \rightarrow$ $W^{1, q}\left(W^{1, r}\right)$ is well defined and analytic. For this purpose, will use the following Lemma.

Lemma 2 For every pair $x_{1}=\left(u_{1}, \sigma_{1}\right), x_{2}=\left(u_{2}, \sigma_{2}\right) \in X$,

$$
b\left(x_{1}, x_{2}\right):=\nabla u_{1} \sigma_{2}+\sigma_{1}\left(\nabla u_{2}\right)^{T} \in W^{1, q}\left(W^{1, r}\right) .
$$

Moreover, the corresponding bilinear mapping $b: X \times X \rightarrow W^{1, q}\left(W^{1, r}\right)$ is continuous, that is, there exists a constant $C$ such that for all $x_{1}, x_{2} \in X$ we have

$$
\left\|b\left(x_{1}, x_{2}\right)\right\|_{W^{1, q}\left(W^{1, r}\right)} \leq C\left\|x_{1}\right\|_{X}\left\|x_{2}\right\|_{X}
$$

Proof Let $x_{1}=\left(u_{1}, \sigma_{1}\right), x_{2}=\left(u_{2}, \sigma_{2}\right) \in X$. Since $r>d, W^{1, r}(\Omega) \subset_{>} L^{\infty}(\Omega)$ so that $W^{1, r}(\Omega)$ is an algebra (see [1]) and there exists a constant $C$ depending only on $\Omega$ such that

$$
\left\|b\left(x_{1}, x_{2}\right)\right\|_{W^{1, r}} \leq C\left\|u_{1}\right\|_{W^{2, r}}\left\|\sigma_{2}\right\|_{W^{1, r}} .
$$

Then we have

$$
\begin{aligned}
\left\|b\left(x_{1}, x_{2}\right)\right\|_{L^{q}\left(W^{1, r}\right)}^{q} & =\int_{0}^{T}\left\|b\left(x_{1}, x_{2}\right)\right\|_{W^{1, r}}^{q} \\
& \leq C \int_{0}^{T}\left\|u_{1}\right\|_{W^{2, r}}^{q}\left\|\sigma_{2}\right\|_{W^{1, r}}^{q} \\
& \leq C\left\|u_{1}\right\|_{L^{\infty}\left(W^{2, r}\right)}^{q} \int_{0}^{T}\left\|\sigma_{2}\right\|_{W^{1, r}}^{q} .
\end{aligned}
$$


Since $q>1, W^{1, q}(0, T) \subset_{>} L^{\infty}(0, T)$ and we also have that $W^{1, q}(B) \subset_{>} L^{\infty}(B)$ for any Banach space $B$, thus

$$
\left\|b\left(x_{1}, x_{2}\right)\right\|_{L^{q}\left(W^{1, r}\right)}^{q} \leq C\left\|u_{1}\right\|_{W^{1, q}\left(W^{2, r}\right)}^{q}\left\|\sigma_{2}\right\|_{L^{q}\left(W^{1, r}\right)}^{q},
$$

which proves that

$$
\left\|b\left(x_{1}, x_{2}\right)\right\|_{L^{q}\left(W^{1, r}\right)} \leq C\left\|u_{1}, \sigma_{1}\right\|_{X}\left\|u_{2}, \sigma_{2}\right\|_{X} .
$$

Similarly, there exists a constant $C$ depending only on $\Omega, \lambda$ and $\eta_{p}$ such that

$$
\begin{aligned}
& \left\|\frac{\partial b}{\partial t}\left(x_{1}, x_{2}\right)\right\|_{L^{q}\left(W^{1, r}\right)}^{q}=\int_{0}^{T}\left\|\frac{\partial b}{\partial t}\left(x_{1}, x_{2}\right)\right\|_{W^{1, r}}^{q} \\
& \quad \leq C \int_{0}^{T}\left(\left\|\frac{\partial u_{1}}{\partial t}\right\|_{W^{2, r}}^{q}\left\|\sigma_{2}\right\|_{W^{1, r}}^{q}+\left\|u_{1}\right\|_{W^{2, r}}^{q}\left\|\frac{\partial \sigma_{2}}{\partial t}\right\|_{W^{1, r}}^{q}\right) \\
& \quad \leq C\left(\left\|\sigma_{2}\right\|_{L^{\infty}\left(W^{1, r}\right)}^{q} \int_{0}^{T}\left\|\frac{\partial u_{1}}{\partial t}\right\|_{W^{2, r}}^{q}+\left\|u_{1}\right\|_{L^{\infty}\left(W^{2, r}\right)}^{q} \int_{0}^{T}\left\|\frac{\partial \sigma_{2}}{\partial t}\right\|_{W^{1, r}}^{q}\right) \\
& \quad \leq C\left(\left\|\sigma_{2}\right\|_{W^{1, q}\left(W^{1, r}\right)}^{q} \int_{0}^{T}\left\|\frac{\partial u_{1}}{\partial t}\right\|_{W^{2, r}}^{q}+\left\|u_{1}\right\|_{W^{1, q}\left(W^{2, r}\right)}^{q} \int_{0}^{T}\left\|\frac{\partial \sigma_{2}}{\partial t}\right\|_{W^{1, r}}^{q}\right),
\end{aligned}
$$

which proves that

$$
\left\|\frac{\partial b}{\partial t}\left(x_{1}, x_{2}\right)\right\|_{L^{q}\left(W^{1, r}\right)} \leq C\left\|u_{1}, \sigma_{1}\right\|_{X}\left\|u_{2}, \sigma_{2}\right\|_{X}
$$

The estimates (25) and (26) prove that $b\left(x_{1}, x_{2}\right) \in W^{1, q}\left(W^{1, r}\right)$ and (24).

Remark 8 In fact we also have proved that $W^{1, q}\left(W^{1, r}\right)$ is an algebra for $1<q<\infty$ and $d<r<\infty$.

Remark 9 For $x \in X$ we have $S(x)=\frac{\lambda}{2 \eta_{p}} b(x, x)$, where $S: X \rightarrow W^{1, q}\left(W^{1, r}\right)$ is introduced in (22). Thus, in virtue of [16], $S$ is well defined and analytic.

Corollary 3 The mapping $F: Y \times X \rightarrow Z$ is well defined and analytic. Moreover, for $x=(v, \tau) \in X$ its Fréchet derivative $D_{x} F(0,0) x$ is given by

$$
D_{x} F(0,0) x=\left(\begin{array}{c}
\frac{\lambda}{2 \eta_{p}} \frac{\partial \tau}{\partial t}+\frac{1}{2 \eta_{p}} \tau-\epsilon(v) \\
\rho \frac{\partial v}{\partial t}+\eta_{s} A_{r} v-P_{r} \operatorname{div} \tau \\
v(., 0) \\
\tau(., 0)
\end{array}\right) .
$$


Proof In order to study the property of the mapping $F: Y \times X \rightarrow Z$ we rewrite it as follows

$$
F(y, x)=L_{1} y+L_{2} x+\left(\begin{array}{c}
\frac{\lambda}{2 \eta_{p}} b(x, x) \\
0 \\
0 \\
0
\end{array}\right)
$$

where $L_{1}: Y \rightarrow Z, L_{2}: X \rightarrow Z$ are bounded linear operator defined for $y=$ $\left(P_{r} f, u_{0}, \sigma_{0}\right) \in Y$ and $x=(u, \sigma) \in X$ by

$$
L_{1} y=\left(\begin{array}{c}
0 \\
-y
\end{array}\right), \quad L_{2} x=\left(\begin{array}{c}
\frac{\lambda}{2 \eta_{p}} \frac{\partial \sigma}{\partial t}+\frac{1}{2 \eta_{p}} \sigma-\epsilon(u) \\
\rho \frac{\partial u}{\partial t}+\eta_{s} A_{r} u-P_{r} \operatorname{div} \sigma \\
u(., 0) \\
\sigma(., 0)
\end{array}\right)
$$

and $b: X \times X \rightarrow W^{1, q}\left(W^{1, r}\right)$ is defined in Lemma 2. Clearly, the first two terms in (27) are analytic. The last term is also analytic in virtue of [16], which proves that $F$ is analytic. Moreover $D_{x} F(0,0)=L_{2}$ which completes the proof.

In order to use the implicit function theorem, it remains to check that $D_{x} F(0,0)$ is an isomorphism from $X$ to $Z$. Therefore, we have to check that, for $g \in W^{1, q}\left(W^{1, r}\right)$ and $\left(h, v_{0}, \tau_{0}\right) \in Y$ there exists a unique $(v, \tau) \in X$ such that

$$
\left\{\begin{aligned}
\frac{\lambda}{2 \eta_{p}} \frac{\partial \tau}{\partial t}+\frac{1}{2 \eta_{p}} \tau-\epsilon(v) & =g, \\
\rho \frac{\partial v}{\partial t}+\eta_{s} A_{r} v-P_{r} \operatorname{div} \tau & =h, \\
v(., 0) & =v_{0}, \\
\tau(., 0) & =\tau_{0} .
\end{aligned}\right.
$$

Lemma 3 Let $d \geq 2$, let $\Omega \subset \mathbb{R}^{d}$ be a bounded,connected open set with boundary of class $\mathcal{C}^{2}$, let $T>0$ and assume $d<r<\infty, 1<q<\infty$. Given $g \in W^{1, q}\left(W^{1, r}\right)$ and $\left(h, v_{0}, \tau_{0}\right) \in Y$, there exists a unique $(v, \tau) \in X$ solution of $(28)$. Moreover, there exists a constant $C$ such that for $g \in W^{1, q}\left(W^{1, r}\right)$ and $\left(h, v_{0}, \tau_{0}\right) \in Y$

$$
\|v, \tau\|_{X} \leq C\left(\|g\|_{W^{1, q}\left(W^{1, r}\right)}+\left\|h, v_{0}, \tau_{0}\right\|_{Y}\right) .
$$

Proof Solving the first equation of (28) we obtain for the extra-stress

$$
\tau=k \tau_{0}+\frac{2 \eta_{p}}{\lambda} k *(\epsilon(v)+g)
$$


with $k \in C^{\infty}([0, T])$ defined by $k(t):=e^{-\frac{t}{\lambda}}$ and the convolution operator $*$ by

$$
(f * g)(t):=\int_{0}^{t} f(t-s) g(s) d s \quad \forall t \in[0, T], \forall f, g \in L^{1}(0, T) .
$$

Introducing (30) in the second equation of (28), yields

$$
\left\{\begin{aligned}
\rho \frac{\partial v}{\partial t}+\eta_{s} A_{r} v+\frac{\eta_{p}}{\lambda} k * A_{r} v & =\tilde{h} \\
v(., 0) & =v_{0} .
\end{aligned}\right.
$$

where $\tilde{h}:=h+P_{r} \operatorname{div}\left(k \tau_{0}\right)+\frac{2 \eta_{p}}{\lambda} P_{r} \operatorname{div}(k * g) \in W^{1, q}\left(\mathcal{H}_{r}\right)$. Since $\Omega$ is of class $\mathcal{C}^{2},-A_{r}$ satisfies the maximal regularity property (see Theorem 15 p. 102 in Sect. 17 of [52] for the $L^{r}\left(L^{r}\right)$ estimate and Remark 13 in Appendix (A) for the $L^{q}\left(L^{r}\right)$ estimate). Moreover, $\overline{\mathcal{D}_{A_{r}}}=L^{r}(\Omega)$ and since $v_{0} \in \mathcal{D}_{A_{r}},-A_{r} v_{0}+\tilde{h}(0) \in E_{1-1 / q, q}$, Corollary 5 and Lemma 14 both in Appendix (A) prove the existence and uniqueness of the solution $v \in W^{2, q}\left(\mathcal{H}_{r}\right) \cap W^{1, q}\left(\mathcal{D}_{A_{r}}\right)$. The estimates of Corollary 5, Lemma 14 and Remark 12 (in Appendix A) ensure the existence of a constant $C$ such that for $\left(h, v_{0}, \tau_{0}\right) \in Y, g \in W^{1, q}\left(W^{1, r}\right)<$

$$
\|v\|_{W^{2, q}\left(L^{r}\right)}+\|v\|_{W^{1, q}\left(\mathcal{D}_{A_{r}}\right)} \leq C\left(\left\|h, v_{0}, \tau_{0}\right\|_{Y}+\|g\|_{W^{1, q}\left(W^{1, r}\right)}\right) .
$$

Because of the regularity of $\Omega$, the graph norm $\|\cdot\|_{\mathcal{D}_{A_{r}}}$ is equivalent to the whole norm $\|\cdot\|_{W^{2, r}}$, thus there exists a constant $C$ such that

$$
\|v\|_{W^{2, q}\left(L^{r}\right)}+\|v\|_{W^{1, q}\left(W^{2, r}\right)} \leq C\left(\left\|h, v_{0}, \tau_{0}\right\|_{Y}+\|g\|_{W^{1, q}\left(W^{1, r}\right)}\right) .
$$

Going back to the extra-stress, Eq. (30), since $g+\epsilon(v) \in W^{1, q}\left(W^{1, r}\right)$, Remark 12 in Appendix (A) ensures that $k *(g+\epsilon(v)) \in W^{2, q}\left(W^{1, r}\right)$ and there exists a constant $C$ such that

$$
\|k *(g+\epsilon(v))\|_{W^{2, q}\left(W^{1, r}\right)} \leq C\left(\|g\|_{W^{1, q}\left(W^{1, r}\right)}+\|v\|_{W^{1, q}\left(W^{2, r}\right)}\right) .
$$

It remains to use (30) to obtain the existence and uniqueness of $\tau \in W^{2, q}\left(W^{1, r}\right)$. Moreover there exists a constant $C$ such that

$$
\|\tau\|_{W^{2, q}\left(W^{1, r}\right)} \leq C\left(\left\|h, v_{0}, \tau_{0}\right\|_{Y}+\|g\|_{W^{1, q}\left(W^{1, r}\right)}\right)
$$

Collecting the estimations (32) and (33) we obtain (29).

Proof (of Theorem 1, part ii) We apply the implicit function theorem to (23). From Corollary 3, F is well defined and analytic, $F(0,0)=0$. Moreover, from Lemma 3 $D_{x} F(0,0)$ is an isomorphism from $X$ to $Z$. Therefore, we can apply the implicit function theorem (see for instance Theorem 4.5 .4 chapter 4 p. 56 of [14]). Thus there 
exists $\delta_{0}>0$ and $\varphi: Y \rightarrow X$ analytic such that for all $y:=\left(P_{r} f, u_{0}, \sigma_{0}\right) \in Y$ with $\|y\|_{Y}<\delta_{0}$ we have $F(y, \varphi(y))=0$.

We will now briefly explain how same arguments can be used to prove parts (i), (iii) and (iv) of Theorem 1.

The proof of part (i) is very similar to the one presented hereabove. Indeed, it suffices to use the spaces

$$
\begin{aligned}
& Y=\left\{\left(P_{r} f, u_{0}, \sigma_{0}\right), \text { such that }\left(f, u_{0}, \sigma_{0}\right) \in L^{q}\left(L^{r}\right) \times E_{1-1 / q, q} \times W^{1, r}\right\}, \\
& X=W^{1, q}\left(\mathcal{H}_{r}\right) \cap L^{q}\left(\mathcal{D}_{A_{r}}\right) \times W^{1, q}\left(W^{1, r}\right), \\
& Z=L^{q}\left(W^{1, r}\right) \times Y
\end{aligned}
$$

and to use Corollary 5 (Appendix A) in order to prove the existence and uniqueness of the function $v$ solution of (31). Concerning part (iii), we shall use the spaces

$$
\begin{aligned}
Y= & \left\{\left(P_{r} f, u_{0}, \sigma_{0}\right), \text { such that }\left(f, u_{0}, \sigma_{0}\right) \in h^{\mu}\left(L^{r}\right) \times \mathcal{D}_{A_{r}} \times W^{1, r}\right. \\
\quad & \text { and } \left.-\eta_{s} A_{r} u_{0}+P_{r} f(0)+P_{r} \operatorname{div} \sigma_{0} \in \overline{\mathcal{D}_{A_{r}}} E_{\mu, \infty}\right\} \\
X= & h^{1+\mu}\left(\mathcal{H}_{r}\right) \cap h^{\mu}\left(\mathcal{D}_{A_{r}}\right) \times h^{1+\mu}\left(W^{1, r}\right), \\
Z= & h^{\mu}\left(W^{1, r}\right) \times Y .
\end{aligned}
$$

and Lemma 13 (Appendix A) in order to prove the existence and uniqueness of the function $v$ solution of (31). Finally, the link between parts (i) and (ii) is the same as between parts (iii) and (iv). Thus we can extend the arguments presented in part (ii) to little Hölder spaces in order to obtain more regularity in time.

\subsection{Proof of Theorem 2}

This result is obtained using the fully nonlinear theory for parabolic problems which can be found in [37]. More precisely, Theorem 8.1.1 pp. 290 will be used on problem (13)-(16) that can be rewritten as follows

$$
\dot{x}(t)=G(t, x(t)), \quad t>0, \quad x(0)=x_{0},
$$

where $x:=(u, \sigma), x_{0}:=\left(u_{0}, \sigma_{0}\right)$ and $G:[0, T] \times \mathcal{D}_{A_{r}} \times W^{1, r} \rightarrow \mathcal{H}_{r} \times W^{1, r}$ is defined by

$$
G(t, x):=\left(\begin{array}{cc}
-\frac{\eta_{s}}{\rho} A_{r} & G_{1} \\
G_{2} & -\frac{1}{\lambda} I_{d}
\end{array}\right) x+\left(\begin{array}{c}
\frac{1}{\rho} P_{r} f(t) \\
\hat{S}(x)
\end{array}\right)
$$

Hereabove, $G_{1} \in \mathcal{L}\left(W^{1, r} ; \mathcal{H}_{r}\right)$ and $G_{2} \in \mathcal{L}\left(\mathcal{D}_{A_{r}}, W^{1, r}\right)$ are defined by

$$
G_{1} \sigma=\frac{1}{\rho} P_{r} \operatorname{div} \sigma, \quad G_{2} u=\frac{2 \eta_{p}}{\lambda} \epsilon(u)
$$


whilst $\hat{S}: \mathcal{D}_{A_{r}} \times W^{1, r} \rightarrow W^{1, r}$ is defined by

$$
\hat{S}(u, \sigma):=(\nabla u) \sigma+\sigma(\nabla u)^{T} .
$$

Lemma 4 The application $\hat{S}: \mathcal{D}_{A_{r}} \times W^{1, r} \rightarrow W^{1, r}$ is well defined and analytic. Moreover, $G:[0, T] \times \mathcal{D}_{A_{r}} \times W^{1, r} \rightarrow \mathcal{H}_{r} \times W^{1, r}$ is continuous with respect to $(t, x)$.

Proof Same arguments as provided in Lemma 2 and Remark 9 in the previous subsection can be used to ensure $\hat{S}: \mathcal{D}_{A_{r}} \times W^{1, r} \rightarrow W^{1, r}$ is well defined and analytic. Let us prove now the continuity of $G$. In order to simplify the notations, let us introduce de linear part of $G$, namely $L \in \mathcal{L}\left(\mathcal{D}_{A_{r}} \times W^{1, r}, \mathcal{H}_{r} \times W^{1, r}\right)$ defined by

$$
L:=\left(\begin{array}{cc}
-\frac{\eta_{s}}{\rho} A_{r} & G_{1} \\
G_{2} & -\frac{1}{\lambda} I_{d}
\end{array}\right)
$$

Fix $(t, x) \in[0, T] \times \mathcal{D}_{A_{r}} \times W^{1, r}$ and let $\left\{t_{n}\right\}_{n \geq 0} \subset[0, T],\left\{x_{n}\right\}_{n \geq 0} \subset \mathcal{D}_{A_{r}} \times W^{1, r}$ such that $t_{n} \rightarrow t$ and $x_{n} \rightarrow x$ when $t$ goes to infinity. Therefore,

$$
\begin{aligned}
& \left\|G(t, x)-G\left(t_{n}, x_{n}\right)\right\|_{L^{r} \times W^{1, r}} \\
& \quad \leq\left\|L\left(x-x_{n}\right)\right\|_{L^{r} \times W^{1, r}}+\left\|\frac{1}{\rho}\left(P_{r} f(t)-P_{r} f\left(t_{n}\right)\right)\right\|_{L^{r}}+\left\|\hat{S}(x)-\hat{S}\left(x_{n}\right)\right\|_{W^{1, r}} .
\end{aligned}
$$

Thus, since $f \in \mathcal{C}^{\mu}\left(L^{r}\right)$ and $\hat{S}$ is continuous from $\mathcal{D}_{A_{r}} \times W^{1, r}$ to $W^{1, r}$, it follows

$$
\begin{aligned}
& \left\|G(t, x)-G\left(t_{n}, x_{n}\right)\right\|_{L^{r} \times W^{1, r}} \\
& \leq\|L\|_{\mathcal{L}\left(\mathcal{D}_{A_{r}} \times W^{1, r}, \mathcal{H}_{r} \times W^{1, r}\right)}\left\|x-x_{n}\right\|_{W^{2, r} \times W^{1, r}} \\
& \quad+C\left(\left\|P_{r} f\right\|_{\mathcal{C}^{\mu}\left(L^{r}\right)}\left|t-t_{n}\right|^{\mu}+\left\|x-x_{n}\right\|_{W^{2, r} \times W^{1, r}}\right) .
\end{aligned}
$$

Hence

$$
\left\|G(t, x)-G\left(t_{n}, x_{n}\right)\right\|_{L^{r} \times W^{1, r}} \rightarrow 0 \text { when } n \rightarrow \infty .
$$

The crucial point in order to prove Theorem 2 is

$$
\left\{\begin{array}{l}
\text { for } t \in[0, T] \text { and } x \in \mathcal{D}_{A_{r}} \times W^{1, r} \text { the Fréchet derivative } D_{x} G(t, x) \\
\text { is the generator of an analytic semigroup. }
\end{array}\right.
$$

The above property will be a consequence of a result by S. B. Angenent [2].

Lemma 5 For $t \in[0, T]$ and $x \in \mathcal{D}_{A_{r}} \times W^{1, r}$ the Fréchet derivative $D_{x} G(t, x)$ is the generator of an analytic semigroup. 
Proof Let $x:=(u, \sigma) \in \mathcal{D}_{A_{r}} \times W^{1, r}$. In order to characterize the Fréchet derivative $D_{x} G(t, x), t \in[0, T]$, let us define the operators $S_{u} \in \mathcal{L}\left(W^{1, r}, W^{1, r}\right)$ and $S_{\sigma} \in \mathcal{L}\left(\mathcal{D}_{A_{r}}, W^{1, r}\right)$ by

$$
S_{u} \tau:=(\nabla u) \tau+\tau \nabla u, \quad \forall \tau \in W^{1, r}
$$

and

$$
S_{\sigma} v:=(\nabla v) \sigma+\sigma \nabla v, \quad \forall v \in \mathcal{D}_{A_{r}} .
$$

Lemma 2 ensures $S_{\sigma} \in \mathcal{L}\left(\mathcal{D}_{A_{r}}, W^{1, r}\right), S_{u} \in \mathcal{L}\left(W^{1, r}, W^{1, r}\right)$. Using this notations we obtain for $t \in[0, T]$ and $x \in \mathcal{D}_{A_{r}} \times W^{1, r}$

$$
D_{x} G(t, x)=\left(\begin{array}{cc}
-\frac{\eta_{s}}{\rho} A_{r} & G_{1} \\
G_{2}+S_{\sigma} & -\frac{1}{\lambda} I_{d}+S_{u}
\end{array}\right)
$$

and since $G_{1} \in \mathcal{L}\left(W^{1, r}, \mathcal{H}_{r}\right), G_{2} \in \mathcal{L}\left(\mathcal{D}_{A_{r}}, W^{1, r}\right)$, we have

$$
D_{x} G(t, x) \in \mathcal{L}\left(\mathcal{D}_{A_{r}} \otimes W^{1, r}, \mathcal{H}_{r} \otimes W^{1, r}\right) .
$$

Finally, since $-A_{r}: \mathcal{D}_{A_{r}} \rightarrow \mathcal{H}_{r}$ is generator of an analytic semigroup (see [32]), Lemma 2.6 p. 98 (part a) of [2] concludes the proof.

Let us go back to the proof of Theorem 2 .

Proof (of Theorem 2) We apply Theorem 8.1.1 p. 290 of [37] with $\bar{u}=x_{0}=\left(u_{0}, \sigma_{0}\right)$, $t_{0}=0, \bar{t}=0$ and $\mathcal{O}=\mathcal{D}_{A_{r}} \times W^{1, r}$. Since $\overline{\mathcal{D}_{A_{r}} \times W^{1, r}}=\mathcal{H}_{r} \times W^{1, r}$, the condition $G\left(0, x_{0}\right) \in \overline{\mathcal{D}_{A_{r}} \times W^{1, r}}$ is satisfied for all $x_{0} \in \mathcal{D}_{A_{r}} \times W^{1, r}$. Thus it remains to check

(i) property (34) is satisfied,

(ii) for $t \in[0, T]$ and $x \in \mathcal{D}_{A_{r}} \times W^{1, r}$, the graph norm of the operator $D_{x} G(t, x)$ is equivalent to the norm $\|\cdot\|_{W^{2, r} \times W^{1, r}}$,

(iii) $(t, x) \mapsto G(t, x)$ is continuous with respect to $(t, x)$, and it is Fréchet differentiable with respect to $x$,

(iv) for all $\bar{x}:=(\bar{u}, \bar{\sigma}) \in \mathcal{D}_{A_{r}} \times W^{1, r}$ there are $R=R(\bar{x}), L=L(\bar{x}), K=K(\bar{x})>$ 0 verifying

$$
\begin{aligned}
& \left\|D_{x} G(t, x)-D_{x} G(t, z)\right\|_{\mathcal{L}\left(\mathcal{D}_{A_{r}} \times W^{1, r}, \mathcal{H}_{r} \times W^{1, r}\right)} \leq L\|x-z\|_{W^{2, r} \times W^{1, r}}, \\
& \quad\|G(t, x)-G(s, x)\|_{L^{r} \times W^{1, r}} \\
& \quad+\left\|D_{x} G(t, x)-D_{x} G(s, x)\right\|_{\mathcal{L}\left(\mathcal{D}_{A_{r}} \times W^{1, r}, \mathcal{H}_{r} \times W^{1, r}\right)} \leq K|t-s|^{\mu},
\end{aligned}
$$

for all $t, s \in[0, T], x, z \in B(\bar{x}, R) \subset \mathcal{D}_{A_{r}} \times W^{1, r}$. 
Relation (i) is satisfied by using Lemma 5. Property (ii) is satisfied since $W^{1, r} \subset_{>} L^{\infty}$ (see [1]). The application $G$ is continuous by Lemma 4. The Fréchet derivative is given by (35) and is well defined. Finally, (iv) may be proved as follow. Let $x=(u, \sigma)$, $z:=(v, \tau)$ and $\tilde{z}:=(w, \xi)$ all belonging to $\mathcal{D}_{A_{r}} \times W^{1, r}$, using again the continuous embedding $W^{1, r} C_{>} L^{\infty}$ we have

$$
\begin{aligned}
& \left\|D_{x} G(t, x) \tilde{z}-D_{x} G(t, z) \tilde{z}\right\|_{L^{r} \times W^{1, r}}=\left\|D_{x} \hat{S}(x) \tilde{z}-D_{x} \hat{S}(z) \tilde{z}\right\|_{L^{r} \times W^{1, r}} \\
& \quad=\left\|\nabla(u-v) \xi+\xi(\nabla(u-v))^{T}+\nabla w(\sigma-\tau)+(\sigma-\tau)(\nabla w)^{T}\right\|_{W^{1, r}} \\
& \quad \leq C\|\tilde{z}\|_{W^{2, r} \times W^{1, r}}\|x-z\|_{W^{2, r} \times W^{1, r}},
\end{aligned}
$$

where $C$ is independent of $u$ and $\sigma$. Moreover, for $t, s \in[0, T]$ and $x \in \mathcal{D}_{A_{r}} \times W^{1, r}$

$$
D_{x} G(t, x)=D_{x} G(s, x) .
$$

Hence, since $f \in \mathcal{C}^{\mu}\left(L^{r}\right)$, we have for $t, s \in[0, T]$ and $x \in \mathcal{D}_{A_{r}} \times W^{1, r}$

$$
\|G(t, x)-G(s, x)\|_{L^{r} \times W^{1, r}}=\left\|\frac{1}{\rho}\left(P_{r} f(t)-P_{r} f(s)\right)\right\|_{L^{r}} \leq C|t-s|^{\mu},
$$

where $C$ is independent of $t, s$ and $x$. Relations (i)-(iv) ensure the existence of $0<T_{*}<T$ such that there exists a solution

$$
x \in \mathcal{C}^{0}\left(\left[0, T_{*}\right], \mathcal{D}_{A_{r}} \times W^{1, r}\right) \cap \mathcal{C}^{1}\left(\left[0, T_{*}\right], \mathcal{H}_{r} \times W^{1, r}\right)
$$

of (13)-(16).

\section{Existence of the finite element approximation and a priori error estimates}

In this section we assume that $\Omega$ is a convex polygon and that

$$
2 \leq q<+\infty, \quad 2=d \leq r<+\infty .
$$

We set

$$
\begin{aligned}
& Y=L^{q}\left(L^{r}\right) \times E_{1-1 / q, q} \times W^{1, r}, \\
& X=W^{1, q}\left(L^{r}\right) \cap L^{q}\left(W^{2, r}\right) \times W^{1, q}\left(W^{1, r}\right),
\end{aligned}
$$

the data and solution spaces, respectively. According to Theorem 1 part (i), Corollary 1 and Remark 7 we know that, if $y=\left(f, u_{0}, \sigma_{0}\right) \in Y$ is sufficiently small, then there exists a unique solution

$$
(u(y), \sigma(y), p(y))
$$


of (1)-(6), the mapping

$$
y \rightarrow(u(y), \sigma(y), p(y))
$$

being analytic (therefore continuous).

In order to prove that the solution of the nonlinear finite element discretization (12) exists and converges to that of (1)-(6), we introduce $X_{h} \subset X$ defined by

$$
X_{h}=L^{2}\left(V_{h}\right) \times L^{\infty}\left(M_{h}\right)
$$

equipped with the norm $\|\cdot\|_{X_{h}}$ defined for all $x_{h}=\left(u_{h}, \sigma_{h}\right) \in X_{h}$ by

$$
\left\|x_{h}\right\|_{X_{h}}^{2}:=2 \eta_{s} \int_{0}^{T}\left\|\epsilon\left(u_{h}(t)\right)\right\|_{L^{2}(\Omega)}^{2} d t+\frac{\lambda}{4 \eta_{p}} \sup _{t \in[0, T]}\left\|\sigma_{h}(t)\right\|_{L^{2}(\Omega)}^{2} .
$$

Then, we rewrite the solution of (12) as the following fixed point problem. Given $y=\left(f, u_{0}, \sigma_{0}\right) \in Y$, find $x_{h}=\left(u_{h}, \sigma_{h}\right) \in X_{h}$ such that

$$
x_{h}=\mathrm{T}_{h}\left(y, S\left(x_{h}\right)\right) \text {, }
$$

where $S$ is still defined as in (22) but has been extended to the larger space

$$
S: L^{2}\left(H^{1}\right) \times L^{\infty}\left(L^{2}\right) \rightarrow L^{2}\left(L^{2}\right) .
$$

The operator $T_{h}$ is the semi-discrete time-dependent three fields Stokes problem defined by

$$
\begin{aligned}
\mathrm{T}_{h}: Y \times L^{2}\left(L^{2}\right) & \rightarrow X_{h} \\
\left(f, u_{0}, \sigma_{0}, g\right) & \rightarrow \mathrm{T}_{h}\left(f, u_{0}, \sigma_{0}, g\right) \underset{\text { def. }}{=}\left(\tilde{u}_{h}, \tilde{\sigma}_{h}\right)
\end{aligned}
$$

where for $t \in(0, T)$

$$
\left(\tilde{u}_{h}, \tilde{\sigma}_{h}, \tilde{p}_{h}\right): t \longmapsto\left(\tilde{u}_{h}(t), \tilde{\sigma}_{h}(t), \tilde{p}_{h}(t)\right) \in V_{h} \times M_{h} \times Q_{h}
$$

satisfies $\tilde{u}_{h}(0)=i_{h} u_{0}, \tilde{\sigma}_{h}(0)=i_{h} \sigma_{0}$ and

$$
\begin{aligned}
& \rho\left(\frac{\partial \tilde{u}_{h}}{\partial t}, v_{h}\right)+2 \eta_{s}\left(\epsilon\left(\tilde{u}_{h}\right), \epsilon\left(v_{h}\right)\right)-\left(\tilde{p}_{h}, \operatorname{div} v_{h}\right)+\left(\tilde{\sigma}_{h}, \epsilon\left(v_{h}\right)\right)-\left(f, v_{h}\right) \\
& +\left(\operatorname{div} \tilde{u}_{h}, q_{h}\right)+\sum_{K \in \mathcal{T}_{h}} \frac{\alpha h_{K}^{2}}{2 \eta_{p}}\left(\nabla \tilde{p}_{h}, \nabla q_{h}\right)_{K} \\
& +\frac{1}{2 \eta_{p}}\left(\tilde{\sigma}_{h}, \tau_{h}\right)+\frac{\lambda}{2 \eta_{p}}\left(\frac{\partial \tilde{\sigma}_{h}}{\partial t}, \tau_{h}\right)-\left(\epsilon\left(\tilde{u}_{h}\right), \tau_{h}\right)-\frac{\lambda}{2 \eta_{p}}\left(g, \tau_{h}\right)=0
\end{aligned}
$$

for all $\left(v_{h}, \tau_{h}, q_{h}\right) \in V_{h} \times M_{h} \times Q_{h}$, a.e in $(0, T)$. 
It should be noticed that, given $y=\left(f, u_{0}, \sigma_{0}\right) \in Y$ sufficiently small, the solution $x(y)=(u(y), \sigma(y)) \in X$ of the continuous Oldroyd-B problem (1)-(6) also satisfies a fixed point problem, namely

$$
x(y)=\mathrm{T}(y, S(x(y))) .
$$

Here the operator $\mathrm{T}$ is the time-dependent three fields Stokes problem defined by

$$
\begin{aligned}
\mathrm{T}: Y \times L^{q}\left(W^{1, r}\right) & \rightarrow X \\
\left(f, u_{0}, \sigma_{0}, g\right) & \rightarrow \mathrm{T}\left(f, u_{0}, \sigma_{0}, g\right) \underset{\text { def. }}{=}(\tilde{u}, \tilde{\sigma}),
\end{aligned}
$$

where $(\tilde{u}, \tilde{\sigma}, \tilde{p})$ satisfy

$$
\begin{aligned}
& \rho \frac{\partial \tilde{u}}{\partial t}-2 \eta_{s} \operatorname{div} \epsilon(\tilde{u})+\nabla \tilde{p}-\operatorname{div} \tilde{\sigma}=f \quad \text { in } \Omega \times(0, T), \\
& \operatorname{div} \tilde{u}=0 \quad \text { in } \Omega \times(0, T), \\
& \frac{1}{2 \eta_{p}} \tilde{\sigma}+\frac{\lambda}{2 \eta_{p}}\left(\frac{\partial \tilde{\sigma}}{\partial t}\right)-\epsilon(\tilde{u})=g \text { in } \Omega \times(0, T), \\
& \tilde{u}(\cdot, 0)=u_{0} \text { in } \Omega \\
& \tilde{\sigma}(\cdot, 0)=\sigma_{0} \text { in } \Omega \\
& \tilde{u}=0 \text { on } \partial \Omega \times(0, T) .
\end{aligned}
$$

We then have the following stability and convergence result, which proof can be found in Appendix (B).

Lemma 6 The operator $\mathrm{T}_{h}$ is well defined and uniformly bounded with respect to $h$ : there exists $C_{1}>0$ such that for all $h>0$ and for all $\left(f, u_{0}, \sigma_{0}, g\right) \in Y \times L^{2}\left(L^{2}\right)$ we have

$$
\left\|\mathrm{T}_{h}\left(f, u_{0}, \sigma_{0}, g\right)\right\|_{X_{h}} \leq C_{1}\left(\left\|f, u_{0}, \sigma_{0}\right\|_{Y}+\|g\|_{L^{2}\left(L^{2}\right)}\right) .
$$

Moreover, there exists $C_{2}>0$ such that for all $h>0$ and for all $\left(f, u_{0}, \sigma_{0}, g\right) \in$ $Y \times L^{q}\left(W^{1, r}\right)$ we have

$$
\left\|\left(\mathrm{T}-\mathrm{T}_{h}\right)\left(f, u_{0}, \sigma_{0}, g\right)\right\|_{X_{h}} \leq C_{2} h\left(\left\|f, u_{0}, \sigma_{0}\right\|_{Y}+\|g\|_{L^{q}\left(W^{1, r}\right)}\right) .
$$

Our goal is now to prove that (36) has a unique solution converging to that of (38). For this purpose, we use, as in [43], an abstract framework and write (36) as the following problem : given $y=\left(f, u_{0}, \sigma_{0}\right) \in Y$, find $x_{h}=\left(u_{h}, \sigma_{h}\right) \in X_{h}$ such that

$$
F_{h}\left(y, x_{h}\right)=0
$$

where $F_{h}: Y \times X_{h} \rightarrow X_{h}$ is defined by

$$
F_{h}\left(y, x_{h}\right)=x_{h}-\mathrm{T}_{h}\left(y, S\left(x_{h}\right)\right) .
$$


In order to prove existence and convergence of a solution to (47), we use Theorem 2.1 of [15]. The mapping $F_{h}: Y \times X_{h} \rightarrow X_{h}$ is $\mathcal{C}^{1}$. Moreover, we need to prove that the scheme is consistent, that $D_{x} F_{h}$ has bounded inverse at $i_{h} x$ - recall that $i_{h}$ is the $L^{2}(\Omega)$ projection onto the finite element space, $x$ is the solution of (38) - and that $D_{x} F_{h}$ is locally Lipschitz at $i_{h} x$.

Lemma 7 Let $\delta_{0}$ be as in Theorem 1 part $\left.i\right), 1$, let $y:=\left(f, u_{0}, \sigma_{0}\right) \in Y$ with $\|y\|_{Y} \leq \delta_{0}$ and let $x(y)=(u(y), \sigma(y)) \in X$ be the solution of (38). Then, there exists a constant $C_{1}$ such that for all $y \in Y$ with $\|y\|_{Y} \leq \delta_{0}$, for all $0<h \leq 1$, we have

$$
\left\|F_{h}\left(y, i_{h} x(y)\right)\right\|_{X_{h}} \leq C_{1} h\left(\|y\|_{Y}+\|x(y)\|_{X}+\|x(y)\|_{X}^{2}\right)
$$

Moreover, there exists a constant $C_{2}$ such that for all $y \in Y$ with $\|y\|_{Y} \leq \delta_{0}$, for all $0<h \leq 1$, for all $z \in X_{h}$ we have

$$
\left\|D_{x} F_{h}\left(y, i_{h} x(y)\right)-D_{x} F_{h}(y, z)\right\|_{\mathcal{L}\left(X_{h}\right)} \leq \frac{C_{2}}{h}\left\|i_{h} x(y)-z\right\|_{X_{h}} .
$$

Proof Using (38) and (48), we have

$$
\begin{aligned}
F_{h}\left(y, i_{h} x\right) & =i_{h} x-x-\mathrm{T}_{h}\left(y, S\left(i_{h} x\right)\right)+\mathrm{T}(y, S(x)) \\
& =i_{h} x-x+\mathrm{T}_{h}\left(0, S(x)-S\left(i_{h} x\right)\right)+\left(\mathrm{T}-\mathrm{T}_{h}\right)(y, S(x)),
\end{aligned}
$$

so that,

$$
\begin{aligned}
& \frac{1}{3}\left\|F_{h}\left(y, i_{h} x\right)\right\|_{X_{h}}^{2} \leq\left\|i_{h} x-x\right\|_{X_{h}}^{2} \\
& \quad+\left\|\mathrm{T}_{h}\left(0, S(x)-S\left(i_{h} x\right)\right)\right\|_{X_{h}}^{2}+\left\|\left(\mathrm{T}-\mathrm{T}_{h}\right)(y, S(x))\right\|_{X_{h}}^{2} .
\end{aligned}
$$

Using standard interpolation results for the first term of the right hand side, Lemma 6 for the second and third terms, it follows that

$$
\begin{aligned}
& \left\|F_{h}\left(y, i_{h} x\right)\right\|_{X_{h}}^{2} \\
& \quad \leq C\left(h^{2}\|x\|_{X}^{2}+\left\|S(x)-S\left(i_{h} x\right)\right\|_{L^{2}\left(L^{2}\right)}^{2}+h^{2}\|y\|_{Y}^{2}+h^{2}\|S(x)\|_{L^{q}\left(W^{1, r}\right)}^{2}\right)
\end{aligned}
$$

$C$ being independent of $h$ and $y$. Proceeding as in Lemma 1, we have

$$
\|S(x)\|_{L^{q}\left(W^{1, r}\right)}^{2} \leq C\|x\|_{X}^{4},
$$


$C$ being independent of $h$ and $y$. On the other hand, we also have

$$
\begin{aligned}
& \frac{2 \eta_{p}}{\lambda}\left(S(x)-S\left(i_{h} x\right)\right) \\
& =\nabla u \sigma+\sigma(\nabla u)^{T}-\left(\nabla i_{h} u\right) i_{h} \sigma-i_{h} \sigma\left(\nabla i_{h} u\right)^{T} \\
& =\nabla\left(u-i_{h} u\right) \sigma+\left(\nabla i_{h} u\right)\left(\sigma-i_{h} \sigma\right) \\
& \quad+\sigma\left(\nabla\left(u-i_{h} u\right)\right)^{T}+\left(\sigma-i_{h} \sigma\right)\left(\nabla i_{h} u\right)^{T},
\end{aligned}
$$

so that, using a Cauchy-Schwarz inequality

$$
\left\|S(x)-S\left(i_{h} x\right)\right\|_{L^{2}\left(L^{2}\right)}^{2} \leq C\left\|x-i_{h} x\right\|_{X_{h}}^{2}\left(\|\sigma\|_{L^{\infty}\left(L^{\infty}\right)}^{2}+\left\|\nabla i_{h} u\right\|_{L^{2}\left(L^{\infty}\right)}^{2}\right),
$$

$C$ being independent of $h$ and $y$. Standard interpolation results lead to

$$
\left\|\nabla i_{h} u\right\|_{L^{\infty}} \leq\|\nabla u\|_{L^{\infty}}+\left\|\nabla\left(i_{h} u-u\right)\right\|_{L^{\infty}} \leq C\|u\|_{W^{2, r}}
$$

$C$ being independent of $h$ and $y$. Thus, using again standard interpolation, we have

$$
\left\|S(x)-S\left(i_{h} x\right)\right\|_{L^{2}\left(L^{2}\right)}^{2} \leq C h^{2}\|x\|_{X}^{4},
$$

$C$ being independent of $h$ and $y$. Finally, (52) and (53) in (51) yields (49).

Let us now prove (50). Let $z=(v, \tau) \in X_{h}$, let $\tilde{z}:=(\tilde{v}, \tilde{\tau}) \in X_{h}$, we have

$$
\left(D_{x} F_{h}\left(y, i_{h} x\right)-D_{x} F_{h}(y, z)\right) \tilde{z}=-\mathrm{T}_{h}\left(0,\left(D S\left(i_{h} x\right)-D S(z)\right) \tilde{z}\right) .
$$

Using Lemma 6 we obtain

$$
\begin{gathered}
\left\|\left(D_{x} F_{h}\left(y, i_{h} x\right)-D_{x} F_{h}(y, z)\right) \tilde{z}\right\|_{X_{h}} \\
\leq C\left\|\left(D S\left(i_{h} x\right)-D S(z)\right) \tilde{z}\right\|_{L^{2}\left(L^{2}\right)},
\end{gathered}
$$

$C$ being independent of $h$ and $y$. We have

$$
\begin{aligned}
& \frac{2 \eta_{p}}{\lambda}\left(D S\left(i_{h} x\right)-D S(z)\right) \tilde{z}=\left(\nabla\left(i_{h} u-v\right)\right) \tilde{\tau}+\tilde{\tau}\left(\nabla\left(i_{h} u-v\right)\right)^{T} \\
& \quad+\nabla \tilde{v}\left(i_{h} \sigma-\tau\right)+\left(i_{h} \sigma-\tau\right)(\nabla \tilde{v})^{T} .
\end{aligned}
$$

Then, using Cauchy-Schwarz inequality, there exists a constant $C$ independent of $h$ and $y$ such that

$$
\begin{aligned}
& \left\|\left(D S\left(i_{h} x\right)-D S(z)\right) \tilde{z}\right\|_{L^{2}\left(L^{2}\right)} \leq C\left(\left\|\nabla\left(i_{h} u-v\right)\right\|_{L^{2}\left(L^{\infty}\right)}\|\tilde{\tau}\|_{L^{\infty}\left(L^{2}\right)}\right. \\
& \left.\quad+\|\nabla \tilde{v}\|_{L^{2}\left(L^{\infty}\right)}\left\|i_{h} \sigma-\tau\right\|_{L^{\infty}\left(L^{2}\right)}\right) .
\end{aligned}
$$


A classical inverse inequality yields

$$
\begin{aligned}
& \left\|\left(D S\left(i_{h} x\right)-D S(z)\right) \tilde{z}\right\|_{L^{2}\left(L^{2}\right)} \leq \frac{C}{h}\left(\left\|\nabla\left(i_{h} u-v\right)\right\|_{L^{2}\left(L^{2}\right)}\|\tilde{\tau}\|_{L^{\infty}\left(L^{2}\right)}\right. \\
& \left.+\|\nabla \tilde{v}\|_{L^{2}\left(L^{2}\right)}\left\|i_{h} \sigma-\tau\right\|_{L^{\infty}\left(L^{2}\right)}\right)
\end{aligned}
$$

so that we finally have

$$
\left\|\left(D S\left(i_{h} x\right)-D S(z)\right) \tilde{z}\right\|_{L^{2}\left(L^{2}\right)} \leq \frac{C}{h}\left\|i_{h} x-z\right\|_{X_{h}}\|\tilde{z}\|_{X_{h}} .
$$

This last inequality in (54) yields (50).

Before proving existence of a solution to (47) we still need to check that $D_{x} F_{h}$ $\left(y, i_{h} x\right)$ is invertible.

Lemma 8 Let $\delta_{0}$ be as in Theorem 1 part $\left.i\right), 1$, let $y:=\left(f, u_{0}, \sigma_{0}\right) \in Y$ with $\|y\|_{Y} \leq \delta_{0}$ and let $x(y)=(u(y), \sigma(y)) \in X$ be the solution of (38). Then, there exists $0<\delta_{1} \leq \delta_{0}$ such that for all $y \in Y$ with $\|y\|_{Y} \leq \delta_{1}$, for all $0<h \leq 1$, we have

$$
\left\|D_{x} F_{h}\left(y, i_{h} x(y)\right)^{-1}\right\|_{\mathcal{L}\left(X_{h}\right)} \leq 2
$$

Proof By definition of $F_{h}$, we have

$$
D_{x} F_{h}\left(y, i_{h} x\right)=I-\mathrm{T}_{h}\left(0, D S\left(i_{h} x\right)\right)
$$

so that we can write

$$
D_{x} F_{h}\left(y, i_{h} x\right)=I-G_{h} \text { with } G_{h}:=\mathrm{T}_{h}\left(0, D S\left(i_{h} x\right)\right) .
$$

If we prove that $\left\|G_{h}\right\|_{\mathcal{L}\left(X_{h}\right)} \leq 1 / 2$ for $y$ sufficiently small, then $D_{x} F_{h}\left(y, i_{h} x\right)$ is invertible and $\left\|D_{x} F_{h}\left(y, i_{h} x\right)^{-1}\right\|_{\mathcal{L}\left(X_{h}\right)} \leq 2$.

Let $z:=(v, \tau) \in X_{h}$. Using Lemma 6 we have

$$
\left\|G_{h}(z)\right\|_{X_{h}} \leq C_{1}\left\|D S\left(i_{h} x\right) z\right\|_{L^{2}\left(L^{2}\right)},
$$

$C_{1}$ being independent of $y$ and $h$. Using the same arguments as in the proof of Lemma 7, we have

$$
\begin{aligned}
\frac{2 \eta_{p}}{\lambda} & \left\|D S\left(i_{h} x\right) z\right\|_{L^{2}\left(L^{2}\right)} \\
& =\left\|\left(\nabla i_{h} u\right) \tau+\tau\left(\nabla i_{h} u\right)^{T}+(\nabla v) i_{h} \sigma+i_{h} \sigma(\nabla v)^{T}\right\|_{L^{2}\left(L^{2}\right)} \\
& \leq 2\left(\left\|\nabla i_{h} u\right\|_{L^{2}\left(L^{\infty}\right)}\|\tau\|_{L^{\infty}\left(L^{2}\right)}+\left\|\nabla i_{h} \sigma\right\|_{L^{\infty}\left(L^{\infty}\right)}\|\nabla v\|_{L^{2}\left(L^{2}\right)}\right) \\
& \leq C_{2}\left(\|u\|_{L^{2}\left(W^{2, r}\right)}\|\tau\|_{L^{\infty}\left(L^{2}\right)}+\|\nabla v\|_{L^{2}\left(L^{2}\right)}\|\sigma\|_{W^{1, q}\left(W^{1, r}\right)}\right)
\end{aligned}
$$


$C_{2}$ being independent of $y$ and $h$. Hence,

$$
\left\|G_{h}(z)\right\|_{X_{h}} \leq C_{3}\|x\|_{X}\|z\|_{X_{h}}
$$

where $C_{3}$ is independent of $y$ and $h$. From Corollary 1 , the mapping $y \rightarrow x(y)$ is continuous, thus if $\|y\|_{Y}$ is sufficiently small we have $\|x\|_{X} \leq 1 /\left(2 C_{3}\right)$ so that

$$
\left\|G_{h}(z)\right\|_{X_{h}} \leq \frac{1}{2}\|z\|_{X_{h}} .
$$

We can now prove existence of a solution to the finite element scheme (12) and convergence to the solution of (1)-(6).

Theorem 3 Let $\delta_{0}$ be as in Theorem 1 part $\left.i\right)$, l, let $y:=\left(f, u_{0}, \sigma_{0}\right) \in Y$ with $\|y\|_{Y} \leq \delta_{0}$ and let $x(y)=(u(y), \sigma(y)) \in X$ be the solution of (38). Then, there exists $0<\delta_{2} \leq \delta_{0}$ and $\zeta>0$ such that for all $y \in Y$ with $\|y\|_{Y} \leq \delta_{2}$, for all $0<h \leq 1$, there exists a unique $x_{h}(y)=\left(u_{h}(y), \sigma_{h}(y)\right)$ in the ball of $X_{h}$ centered at $i_{h} x(y)$ with radius $\zeta h$, satisfying

$$
F_{h}\left(y, x_{h}(y)\right)=0 .
$$

Moreover, the mapping $y \rightarrow x_{h}(y)$ is continuous and there exists $C>0$ independent of $h$ and $y$ such that the following a priori error estimate holds

$$
\left\|x(y)-x_{h}(y)\right\|_{X_{h}} \leq C h .
$$

Remark 10 The above Theorem still holds when the stabilization term in (12) is replaced by

$$
\sum_{K \in \mathcal{T}_{h}} \frac{\alpha h_{K}^{2}}{2 \eta_{p}}\left(-\operatorname{div}\left(2 \eta_{s} \epsilon\left(u_{h}\right)+\sigma_{h}\right)+\nabla p_{h}-f, \nabla q_{h}\right)_{K},
$$

provided $0<\alpha \leq C_{I}$. Here $C_{I}$ is the largest constant satisfying the following inverse estimate

$$
C_{I} \sum_{K \in \mathcal{T}_{h}} h_{K}^{2}\left\|\operatorname{div} \tau_{h}\right\|_{L^{2}(K)}^{2} \leq\left\|\tau_{h}\right\|_{L^{2}(\Omega)}^{2} \quad \forall \tau_{h} \in M_{h} .
$$

Remark 11 Theorem 3 also holds when $y=\left(f, u_{0}, \sigma_{0}\right) \in Y$ is sufficiently small, with $Y$ corresponding to Theorem 1 part (iii) thus defined by

$$
\begin{aligned}
Y= & \left\{\left(f, u_{0}, \sigma_{0}\right) \in h^{\mu}\left(L^{r}\right) \times \mathcal{D}_{A_{r}} \times W^{1, r}\right. \\
& \text { such that } \left.-\eta_{s} A_{r} u_{0}+P_{r} f(0)+P_{r} \operatorname{div} \sigma_{0} \in E_{1-1 / q, q}\right\} .
\end{aligned}
$$

This convergence result in little Hölder spaces rather than Sobolev (with respect to the time variable) will be used in a forthcoming paper [7] when considering a finite element scheme for the Hookean dumbbells model which is a stochastic model formally equivalent to Oldroyd-B. 
In order to prove the above Theorem, we will use the following abstract result.

Lemma 9 (Theorem 2.1 of [15]) Let $Y$ and $Z$ be two real Banach spaces with norms $\|.\|_{Y}$ and $\left\|_{.}\right\|_{Z}$ respectively. Let $G: Y \rightarrow Z$ be $a \mathcal{C}^{1}$ mapping and $v \in Y$ be such that $D G(v) \in \mathcal{L}(Y ; Z)$ is an isomorphism. We introduce the notations

$$
\begin{gathered}
\epsilon=\|G(v)\|_{Z}, \\
\gamma=\left\|D G(v)^{-1}\right\|_{\mathcal{L}(Y ; Z)}, \\
L(\alpha)=\sup _{x \in \bar{B}(v, \alpha)}\|D G(v)-D G(x)\|_{\mathcal{L}(Y ; Z)},
\end{gathered}
$$

with $\bar{B}(v, \alpha)=\left\{y \in Y ;\|v-y\|_{Y} \leq \alpha\right\}$, and we are interested in finding $u \in Y$ such that

$$
G(u)=0 .
$$

We assume that $2 \gamma L(2 \gamma \epsilon) \leq 1$. Then Problem (56) has a unique solution $u$ in the ball $\bar{B}(v, 2 \gamma \epsilon)$ and, for all $x \in \bar{B}(v, 2 \gamma \epsilon)$, we have

$$
\|x-u\|_{Y} \leq 2 \gamma\|G(x)\|_{Z} .
$$

Proof (Proof of Theorem 3) We apply Lemma 9 with $Y=X_{h}, Z=X_{h}, G=F_{h}$ and $v=i_{h} x(y)$. According to Lemma 7 there exists a constant $C_{1}$ independent of $y$ and $h$ such that

$$
\epsilon=\left\|F_{h}\left(y, i_{h} x(y)\right)\right\|_{X_{h}} \leq C_{1} h\left(\|y\|_{Y}+\|x(y)\|_{X}+\|x(y)\|_{X}^{2}\right) .
$$

According to Lemma 8, for $\|y\|_{Y}$ sufficiently small

$$
\gamma=\left\|D_{x} F_{h}\left(y, i_{h} x(y)\right)\right\|_{\mathcal{L}\left(X_{h}\right)} \leq 2 .
$$

According to Lemma 7, there is a constant $C_{2}$ independent of $y$ and $h$ such that

$$
L(\beta)=\sup _{z \in \bar{B}\left(i_{h} x(y), \beta\right)}\left\|D F_{h}\left(i_{h} x(y)\right)-D F_{h}(z)\right\|_{\mathcal{L}\left(X_{h}\right)} \leq \frac{C_{2}}{h} \beta .
$$

Hence, we have

$$
\begin{aligned}
2 \gamma L(2 \gamma \epsilon) & \leq 2.2 \frac{C_{2}}{h}\left(2.2 C_{1} h\left(\|y\|_{Y}+\|x(y)\|_{X}+\|x(y)\|_{X}^{2}\right)\right) \\
& =16 C_{1} C_{2}\left(\|y\|_{Y}+\|x(y)\|_{X}+\|x(y)\|_{X}^{2}\right) .
\end{aligned}
$$

Using the continuity of the mapping $y \rightarrow x(y)$, there exists $0<\delta_{2} \leq \delta_{0}$ such that for all $y \in Y$ with $\|y\|_{Y} \leq \delta_{2}$, then

$$
\|y\|_{Y}+\|x(y)\|_{X}+\|x(y)\|_{X}^{2} \leq \frac{1}{32 C_{1} C_{2}}
$$


so that $2 \gamma L(2 \gamma \epsilon) \leq 1 / 2<1$ and Lemma 9 applies. There exists a unique $x_{h}(y)$ in the ball $\bar{B}\left(i_{h} x(y), 2 \gamma \epsilon\right)$ such that

$$
F_{h}\left(y, x_{h}(y)\right)=0
$$

and we have

$$
\begin{aligned}
& \left\|i_{h} x(y)-x_{h}(y)\right\|_{X_{h}} \leq 4 C_{1} h\left(\|y\|_{Y}+\|x(y)\|_{X}+\|x(y)\|_{X}^{2}\right) \\
& \quad \leq \frac{4 C_{1} h}{32 C_{1} C_{2}}=\frac{1}{8 C_{2}} h .
\end{aligned}
$$

It suffices to use the triangle inequality

$$
\left\|x(y)-x_{h}(y)\right\|_{X_{h}} \leq\left\|x(y)-i_{h} x(y)\right\|_{X_{h}}+\left\|i_{h} x(y)-x_{h}(y)\right\|_{X_{h}}
$$

and standard interpolation results to obtain (55). The fact that the mapping $y \rightarrow x_{h}(y)$ is continuous is a direct consequence of the implicit function theorem used to prove Lemma 9.

Acknowledgments AB thanks Claude Lebris and Tony Lelièvre for interesting discussions during a short stay at CERMICS, Ecole Nationale des Ponts et Chaussées, Paris. PC acknowledges Tudor Ratiu for an invitation to the Bernoulli Center, Lausanne. AB and MP thank Alexei Lozinski and Jacques Rappaz for the permanent interest they have shown for this research.

\section{A Appendix: pertubated abstract Cauchy problem}

The aim of this appendix is to state some existence results taken from [18-20,48-50] and used in this paper on the abstract Cauchy problem

$$
\dot{u}(t)=A u(t)+f(t) \quad t \in(0, T), \quad u(0)=u_{0} .
$$

where $f:[0, T] \rightarrow E, u_{0} \in E$ and $(E,\|\|$.$) is a real Banach space. Then, these$ results will be extended to the case when a convolution term $k * A u$ is added to (57). Here the convolution product $*$ is defined for $f, g \in L^{1}(0, T)$ by

$$
(f * g)(t)=\int_{0}^{t} f(t-s) g(s) d s .
$$

Remark 12 From [48] we have: if $f \in L^{q}(0, T ; E), g \in L^{1}(0, T)$ then $f * g \in$ $L^{q}(0, T ; E)$ and

$$
\|f * g\|_{L^{q}(E)} \leq\|g\|_{L^{1}(0, T)}\|f\|_{L^{q}(E)} .
$$

We have the following result in Hölder spaces (see $[18,19]$ ). 
Theorem 4 Let $(E,\|\|$.$) be a Banach space. Let A be a closed, densely defined opera-$ tor in $E$ with domain $\mathcal{D}_{A_{r}}$, generator of an analytic semigroup on E. Let $0<\mu<1$ and let $f \in \mathcal{C}^{\mu}(E)$. Assume that the compatibility conditions $u_{0} \in \mathcal{D}_{A_{r}}$ and $A u_{0}+f(0) \in$ $E_{\mu, \infty}:=\left(E, \mathcal{D}_{A_{r}}\right)_{\mu, \infty}$ holds. Then there exists a unique solution u of problem (57) in $\mathcal{C}^{1+\mu}(E) \cap \mathcal{C}^{\mu}\left(\mathcal{D}_{A_{r}}\right)$ and $u$ satisfies

$$
u(t)=e^{t A} u_{0}+\int_{0}^{t} e^{(t-s) A} f(s) d s, \quad t \in(0, T) .
$$

Moreover there exists a constant $C$ such that

$$
\|\dot{u}\|_{C^{\mu}(E)}+\|A u\|_{C^{\mu}(E)} \leq C\left(\|f-f(0)\|_{C^{\mu}(E)}+\left\|A u_{0}+f(0)\right\|_{E_{\mu, \infty}}\right) .
$$

A similar result also holds in little Hölder spaces (see again $[18,19])$.

Theorem 5 Let $\left(E,\|.\|_{E}\right)$ be a Banach space. Let A be a closed, densely defined operator in $E$ with domain $\mathcal{D}_{A_{r}}$, generator of an analytic semigroup on $E$. Let $0<$ $\mu<1$ and let $f \in h^{\mu}(E)$. Assume the compatibility conditions $u_{0} \in \mathcal{D}_{A_{r}}$ and $A u_{0}+f(0) \in \overline{\mathcal{D}}_{A_{r}} E_{\mu, \infty}$ where $E_{\mu, \infty}:=\left(E, \mathcal{D}_{A_{r}}\right)_{\mu, \infty}$. Then there exists a unique solution u of problem (57) in $h^{1+\mu}(E) \cap h^{\mu}\left(\mathcal{D}_{A_{r}}\right)$ and u satisfies

$$
u(t)=e^{t A} u_{0}+\int_{0}^{t} e^{(t-s) A} f(s) d s, \quad t \in(0, T) .
$$

Moreover there exists a constant $C$ such that

$$
\|\dot{u}\|_{h^{\mu}(E)}+\|A u\|_{h^{\mu}(E)} \leq C\left(\|f-f(0)\|_{h^{\mu}(E)}+\left\|A u_{0}+f(0)\right\|_{\overline{\mathcal{D}}_{A_{r}}} E_{\mu, \infty}\right) .
$$

Before stating some existence results for Sobolev spaces, we have to introduce the maximal regularity property (MRp).

Definition 1 Let $1<q<\infty$. The operator $A$ possesses the maximal $L^{q}$-regularity property (MRp) if for $u_{0}=0$ and any $f \in L^{q}(E)$, there exists a unique solution $u$ of (57) in $W^{1, q}(E) \cap L^{q}\left(\mathcal{D}_{A}\right)$ and there exists a constant $C$ such that

$$
\|\dot{u}\|_{L^{q}(E)}+\|A u\|_{L^{q}(E)} \leq C\|f\|_{L^{q}(E)} .
$$

Remark 13 Let $1<q_{0}<\infty$. If $A$ possesses the maximal $L^{q_{0}}$-regularity property, then $\beta A+\omega I$ possesses the maximal $L^{q}$-regularity property for all $1<q<\infty$, for all $\beta>0$ and for all $w \in \mathbb{R}$ (see [20,50]).

In general, a maximal regularity result does not hold for Sobolev spaces. It has to be assumed. The following result can be found in [19]. 
Lemma 10 Let $\left(E,\|.\|_{E}\right)$ be a Banach space. Let $A$ be a closed, densely defined operator in $E$ with domain $\mathcal{D}_{A}$, generator of an analytic semigroup $e^{t A}$ on $E$. Let $1<$ $q<\infty$, assume A satisfies MRp, $f \in L^{q}(E)$ and $u_{0} \in E_{1-1 / q, q}:=\left(E, \mathcal{D}_{A}\right)_{1-\frac{1}{q}, q}$. Then there exists a unique solution $u$ of problem $(57)$ in $W^{1, q}(E) \cap L^{q}\left(\mathcal{D}_{A}\right)$ and $u$ satisfies

$$
u(t)=e^{t A} u_{0}+\int_{0}^{t} e^{(t-s) A} f(s) d s, \quad t \in(0, T) .
$$

Moreover, there exists a constant $C$ such that

$$
\|\dot{u}\|_{L^{q}(E)}+\|A u\|_{L^{q}(E)} \leq C\left(\left\|u_{0}\right\|_{E_{1-1 / q, q}}+\|f\|_{L^{q}(E)}\right) .
$$

Assuming more regularity of the data with respect to the time variable, and compatibility conditions at initial time, the following regularity result can be obtained.

Corollary 4 Let $\left(E,\|.\|_{E}\right)$ be a Banach space. Let A be a closed, densely defined operator in $E$ with domain $\mathcal{D}_{A}$, generator of an analytic semigroup on $E$. Let $1<q<\infty$, assume A satisfies MRp, let $f \in W^{1, q}(E)$ and $u_{0} \in E$. Assume that the compatibility conditions $u_{0} \in \mathcal{D}_{A}$ and $A u_{0}+f(0) \in E_{1-1 / q, q}:=\left(E, \mathcal{D}_{A}\right)_{1-\frac{1}{q}, q}$ hold. Then the solution $u$ of (57) satisfies $\dot{u} \in W^{1, q}(E) \cap L^{q}\left(\mathcal{D}_{A}\right)$. Moreover, there exists a constant C such that

$$
\|\ddot{u}\|_{L^{q}(E)}+\|A \dot{u}\|_{E} \leq C\left(\left\|A u_{0}+f(0)\right\|_{E_{1-1 / q, q}}+\|\dot{f}\|_{L^{q}(E)}\right) .
$$

We now perturb the first equation of (57) by adding a term of the form $k * A u$. In order to obtain an existence result, this following technical Lemma will be useful.

Lemma 11 Given $\beta \neq 0, m \geq 1$ and $k \in W^{m, 1}(0, T)$, there exists an unique $b \in$ $W^{m+1,1}(0, T)$ such that

$$
\beta b+k * b=1 .
$$

Proof We recall a result given in [44], Theorem 1.4 p.46. For all $p \geq 1$, there exists an unique $r: W^{m, p}(0, T) \rightarrow W^{m, p}(0, T)$ such that for all $a \in W^{m, p}(0, T)$

$$
r(a)+a * r(a)=a .
$$

Then, taking $a=\beta^{-1} k$ in the equation above, the unique solution $S\left(b_{0}\right) \in W^{m, 1}(0, T)$ such that $S\left(b_{0}\right)+\beta^{-1} k * S\left(b_{0}\right)=1$ is given by

$$
S\left(b_{0}\right)=1-r\left(\beta^{-1} k\right) * 1 .
$$

Thus we obtain

$$
b(t)=\beta^{-1} S\left(b_{0}\right)=\beta^{-1}-\beta^{-1} r\left(\beta^{-1} k\right) * 1
$$


and

$$
\dot{b}(t)=-\beta^{-1} r\left(\beta^{-1} k\right) \text { in } L^{1}(0, T)
$$

Since $r\left(\beta^{-1} k\right) \in W^{m, 1}(0, T)$, it follows $b \in W^{m+1,1}(0, T)$.

Lemma 12 Let $\left(E,\|.\|_{E}\right)$ be a Banach space. Let A be a closed, densely defined operator in $E$ with domain $\mathcal{D}_{A}$, generator of an analytic semigroup on $E$. Let $1<q<\infty$, assume $A$ satisfies MRp, let $\beta>0, \gamma \in \mathbb{R}$ and $a \in L^{1}(0, T)$. Let $f \in L^{q}(E), u_{0} \in E_{1-1 / q, q}:=\left(E, \mathcal{D}_{A}\right)_{1-\frac{1}{q}, q}$, then there exists a unique $u \in$ $W^{1, q}(E) \cap L^{q}\left(\mathcal{D}_{A}\right)$ satisfying

$$
\dot{u}=\beta A u+\gamma u+a * u+f, \quad u(0)=u_{0} .
$$

Moreover, there exists a constant $C$ such that

$$
\|\dot{u}\|_{L^{q}(E)}+\|A u\|_{L^{q}(E)} \leq C\left(\|f\|_{L^{q}(E)}+\left\|u_{0}\right\|_{E_{1-1 / q, q}}\right) .
$$

Proof Let $B:=\beta A+\gamma$, since $A$ satisfies the MRp using Remark 13 it follows $B$ satisfies the MRp and there exists a constant such that

$$
\|A u\|_{L^{q}(E)}+\|u\|_{L^{q}(E)} \leq C\left(\|B u\|_{L^{q}(E)}+\|u\|_{L^{q}(E)}\right)
$$

for all $u \in \mathcal{D}_{A}$. Therefore, it remains to prove for given $f \in L^{q}(E)$ and $u_{0} \in E_{1-1 / q, q}$ there exists an unique $z \in W^{1, q}(E) \cap L^{q}\left(\mathcal{D}_{A}\right)$ such that

$$
z=e^{t B} u_{0}+\int_{0}^{t} e^{(t-s) B} f(s) d s+\int_{0}^{t} e^{(t-s) B} a * z(s) d s .
$$

Lemma 10 ensures $z_{0}:=e^{t B} u_{0}+\int_{0}^{t} e^{(t-s) B} f(s) d s \in W^{1, q}(E) \cap L^{q}\left(\mathcal{D}_{A}\right)$. We rewrite (60) as a fixed point problem. Given $z_{0} \in Z:=L^{q}(E)$ and let $F: Z \rightarrow Z$ defined for all $z \in Z$ by

$$
F(z):=v
$$

where, $v \in Z$ satisfies

$$
\dot{v}=B v+a * z, \quad v(0)=0 .
$$

Let us notice $F$ is well defined using Remark 12 and Lemma 10. Then (60) becomes

$$
z=z_{0}+F(z)
$$

We will show there exists $n>0$ such that

$$
\left\|F^{n}\right\|_{\mathcal{L}(Z)}<1
$$


Lemma 10 again ensures there exists a constant $C$ such that

$$
\|v\|_{L^{q}(E)} \leq C\|a * u\|_{L^{q}(E)} \leq C\|a\|_{L^{1}(0, T)}\|u\|_{L^{q}(E)} .
$$

Denoting by $c^{(n)}:=\underbrace{c * \ldots * c}_{n \text { times }}$ for all $c \in L^{1}(0, T)$, it follows

$$
\left\|F^{n}\right\|_{\mathcal{L}(Z)} \leq C^{n}\left\|a^{(n)}\right\|_{L^{1}(0, T)}
$$

Since

$$
\|c * c\|_{L^{1}(0, T)} \leq\|c\|_{L^{\infty}(0, T)} *\|c\|_{L^{\infty}(0, T)}=\|c\|_{L^{\infty}(0, T)}^{2} 1 * 1=\|c\|_{L^{\infty}(0, T)}^{2} T,
$$

for all $c \in L^{\infty}(0, T)$ we find

$$
\left\|c^{(n)}\right\|_{L^{1}(0, T)} \leq\|c\|_{L^{\infty}(0, T)}^{n} \frac{T^{n-1}}{(n-1) !}, \quad \forall c \in L^{\infty}(0, T) .
$$

Using the above inequality in (62), it follows

$$
\left\|F^{n}\right\|_{\mathcal{L}(Z)} \leq \frac{T^{n-1}}{(n-1) !} C^{n}\|a\|_{L^{\infty}(0, T)}^{n}
$$

which tends to 0 when $n$ goes to infinity. Thus (61) is proved and a fixed point theorem (see Theorem 4.4.1 of [16]) ensures the existence of an unique $z \in Z$ satisfying (60) and there exists a constant constant $C$ such that

$$
\|z\|_{L^{q}(E)} \leq C\left\|z_{0}\right\|_{L^{q}(E)}
$$

The fact that $z \in W^{1, q}(E) \cap L^{q}(E)$ is a direct consequence of (60) since $z_{0}, a * z \in$ $W^{1, q}(E) \cap L^{q}(E)$. It remains to prove the estimation (58). Going back to (60) and using Lemma 10 again, there exists a constant $C$ such that

$$
\begin{aligned}
& \|\dot{z}\|_{L^{q}(E)}+\|B u\|_{L^{q}(E)} \\
& \quad \leq C\left(\left\|u_{0}\right\|_{E_{1-1 / q, q}}+\|f\|_{L^{q}(E)}+\|a * u\|_{L^{q}(E)}\right) .
\end{aligned}
$$

Using (63) we obtain

$$
\|a * z\|_{L^{q}(E)} \leq\|a\|_{L^{1}(0, T)}\|z\|_{L^{q}(E)} \leq C\|a\|_{L^{1}(0, T)}\left\|z_{0}\right\|_{L^{q}(E)},
$$

which coupled with (59) and (64) proves (70).

Corollary 5 Let $\left(E,\|.\|_{E}\right)$ be a Banach space. Let A be a closed, densely defined operator in $E$ with domain $\mathcal{D}_{A}$, generator of an analytic semigroup on $E$. Let $1<$ $q<\infty$, assume A satisfies MRp, let $\beta>0, k \in W^{1,1}(0, T), f \in L^{q}(E)$ and 
$u_{0} \in E_{1-1 / q, q}:=\left(E, \mathcal{D}_{A}\right)_{1-\frac{1}{q}, q}$, then there exists a unique $u \in W^{1, q}(E) \cap L^{q}\left(\mathcal{D}_{A}\right)$ satisfying

$$
\dot{u}=\beta A u+k * A u+f, \quad u(0)=u_{0} .
$$

Moreover, there exists a constant $C$ such that

$$
\|\dot{u}\|_{L^{q}(E)}+\|A u\|_{L^{q}(E)} \leq C\left(\|f\|_{L^{q}(E)}+\left\|u_{0}\right\|_{E}\right) .
$$

Proof Since $k \in W^{1,1}(0, T)$, Lemma 11 ensures the existence of a $b \in W^{2,1}(0, T)$ such that

$$
\beta b+k * b=1
$$

Moreover $b(0)=\beta^{-1}$. Convolving the equation for $u$ in (65) and using the equation above, we have

$$
b * \dot{u}=1 * A u+b * f .
$$

Differentiating with respect to time the equation above, using $b(0)=\beta^{-1}$, we obtain

$$
\beta^{-1} \dot{u}+\dot{b} * \dot{u}=A u+\beta^{-1} f+\dot{b} * f .
$$

Noticing that

$$
\dot{b} * \dot{u}+\dot{b} u_{0}=\frac{d}{d t}(\dot{b} * u)=\dot{b}(0) u+\ddot{b} * u,
$$

the equation (68) becomes

$$
\dot{u}=\beta A u-\beta \dot{b}(0) u+f+\beta \dot{b} * f+\beta \dot{b} u_{0}-\beta \ddot{b} * u .
$$

Differentiating equation (67) and since $\dot{b} \in \mathcal{C}^{0}([0, T]), k \in W^{1,1}(0, T)$ we find $\dot{b}(0)=-\beta^{-2} k(0)$. Finally, (65) reduce to

$$
\dot{u}=\beta A u+f+\beta \dot{b} * f+\beta \dot{b} u_{0}-\beta \ddot{b} * u+\beta^{-1} k(0) u, \quad u(0)=u_{0} .
$$

The Lemma 12 completes the proof.

The previous Corollary also holds in little Hölder spaces.

Lemma 13 Let $\left(E,\|.\|_{E}\right)$ be a Banach space. Let A be a closed, densely defined operator in $E$ with domain $\mathcal{D}_{A_{r}}$, generator of an analytic semigroup on E. Let $0<\mu<1$. Let $k \in W^{1, \frac{1}{1-\mu}}(0, T), f \in h^{\mu}([0, T] ; E)$ and $u_{0} \in \mathcal{D}(A)$ satisfying $A u_{0}+f(0) \in$ $\overline{\mathcal{D}(A)}\left(E, \mathcal{D}_{A}\right)_{\mu, \infty}$, then there exists a unique $u \in h^{1+\mu}([0, T] ; E) \cap h^{\mu}([0, T] ; \mathcal{D}(A))$ satisfying

$$
\dot{u}=\beta A u+k * A u+f, \quad u(0)=u_{0} .
$$


Proof The proof use same arguments of the proof for Corollary 5. It has to be slightly modified in the two following senses. Remark 12 , has be replace by the affirmation: let $0<\mu<1$, for all $g \in L^{\frac{1}{1-\mu}}(0, T)$, for all $f \in h^{\mu}([0, T] ; E)$ there exists a constant $C$ such that

$$
\|f * g\|_{h^{\mu}(E)} \leq C\|g\|_{L^{\frac{1}{1-\mu}}(0, T)}\|f\|_{h^{\mu}(E)} .
$$

In the proof for Lemma 12, relation (62) does not holds in $h^{\kappa}(E)$ but using some properties of the operator $L \in \mathcal{L}\left(h^{\mu}(E)\right)$ defined for $v \in h^{\mu}(E)$ by $L v:=a * v$ and Theorem 3 p.211 and Theorem 4 p. 212 in [53] the same conclusion follows.

Lemma 14 Let $\left(E,\|.\|_{E}\right)$ be a Banach space. Let A be a closed, densely defined operator in $E$ with domain $\mathcal{D}_{A}$, generator of an analytic semigroup on $E$. Let $1<q<\infty$, assume A satisfies MRp, let $\beta>0$ and $k \in W^{1,1}(0, T)$. Let $f \in W^{1, q}(E), u_{0} \in \mathcal{D}_{A}$ such that $\beta A u_{0}+f(0) \in E_{1-1 / q, q}:=\left(E, \mathcal{D}_{A}\right)_{1-\frac{1}{q}, q}$, then there exists a unique $u \in W^{2, q}(E) \cap W^{1, q}\left(\mathcal{D}_{A}\right)$ satisfying

$$
\dot{u}=\beta A u+k * A u+f, \quad u(0)=u_{0} .
$$

Moreover, there exists a constant $C$ such that

$$
\begin{aligned}
& \|\ddot{u}\|_{L^{q}(E)}+\|A \dot{u}\|_{L^{q}(E)} \\
& \quad \leq C\left(\|\dot{f}\|_{L^{q}(E)}+\left\|A u_{0}\right\|_{E}+\left\|A u_{0}+f(0)\right\|_{E_{1-1 / q, q}}\right) .
\end{aligned}
$$

Proof Let $u$ be the unique solution in $W^{1, q}(E) \cap L^{q}\left(\mathcal{D}_{A}\right)$ satisfying

$$
\dot{u}=\beta A u+k * A u+f, \quad u(0)=u_{0} .
$$

Let us define $z \in W^{1, q}(E) \cap L^{q}\left(\mathcal{D}_{A}\right)$ such that

$$
\dot{z}=\beta A z+k A u_{0}+k * A z+\dot{f} \quad z(0)=\beta A u_{0}+f(0) .
$$

Corollary 5 ensures $z$ is well defined since $\dot{f} \in L^{q}(E)$ and since $\beta A u_{0}+f(0) \in$ $E_{1-1 / q, q}$. Moreover there exists a constant $C$ such that

$$
\begin{aligned}
& \|\dot{z}\|_{L^{q}(E)}+\|A z\|_{L^{q}(E)} \\
& \quad \leq C\left(\left\|A u_{0}+f(0)\right\|_{E_{1-1 / q, q}}+\|\dot{f}\|_{L^{q}(E)}+\left\|k A u_{0}\right\|_{L^{q}(E)}\right) .
\end{aligned}
$$

Let $v \in W^{2, q}(E) \cap W^{1, q}\left(\mathcal{D}_{A}\right)$ defined by

$$
v(t):=u_{0}+\int_{0}^{t} z(s) d s .
$$


We will show $v=u$. Let us recall that if $A$ is a bounded operator we have

$$
\int_{0}^{t} A y(s) d s=A \int_{0}^{t} y(s) d s, \quad \forall y \in L^{1}\left(0, t^{\prime} ; \mathcal{D}_{A}\right), \forall t^{\prime} \in[0, t] .
$$

From (71) and since $A$ is bounded, it follows

$$
\begin{aligned}
z(t)= & \beta A u_{0}+f(0)+\beta A \int_{0}^{t} z(s) d s+f(t)-f(0) \\
& +1 * k A u_{0}+k * A \int_{0}^{T} z(s) d s .
\end{aligned}
$$

Using the definition of $v$ (73) we have

$$
\left\{\begin{aligned}
\dot{v} & =\beta A\left(v-u_{0}\right)+\beta A u_{0}+f(t)+(1 * k) A u_{0}+k * A\left(v-u_{0}\right), \\
v_{0} & =u_{0}
\end{aligned}\right.
$$

Thus the uniqueness of the solution ensured by Corollary 5 proves $v=u$ or $z=\dot{u}$. The estimate (70) is a direct consequence of (72).

\section{B Appendix: Proof of Lemma 6}

A priori, (37) has to be understood in a weak sense with respect to the time variable: find $\left(\tilde{u}_{h}, \tilde{\sigma}_{h}, \tilde{q}_{h}\right) \in X_{h}$ such that

$$
\begin{aligned}
& -\rho \int_{0}^{T}\left(\tilde{u}_{h}, \frac{\partial v_{h}}{\partial t}\right)+\rho\left(u_{0}, v_{h}(0)\right)+2 \eta_{s} \int_{0}^{T}\left(\epsilon\left(\tilde{u}_{h}\right), \epsilon\left(v_{h}\right)\right) \\
& -\int_{0}^{T}\left(\tilde{p}_{h}, \operatorname{div} v_{h}\right)+\int_{0}^{T}\left(\tilde{\sigma}_{h}, \epsilon\left(v_{h}\right)\right)-\int_{0}^{T}\left(f, v_{h}\right) \\
& +\int_{0}^{T}\left(\operatorname{div} \tilde{u}_{h}, q_{h}\right)+\sum_{K \in \mathcal{T}_{h}} \frac{\alpha h_{K}^{2}}{2 \eta_{p}} \int_{0}^{T}\left(\nabla \tilde{p}_{h}, \nabla q_{h}\right)_{K} \\
& +\frac{1}{2 \eta_{p}} \int_{0}^{T}\left(\tilde{\sigma}_{h}, \tau_{h}\right)-\frac{\lambda}{2 \eta_{p}} \int_{0}^{T}\left(\tilde{\sigma}_{h}, \frac{\partial \tau_{h}}{\partial t}\right)+\frac{\lambda}{2 \eta_{p}}\left(\sigma_{0}, \tau_{h}(0)\right) \\
& -\left(\epsilon\left(\tilde{u}_{h}\right), \tau_{h}\right)-\frac{\lambda}{2 \eta_{p}} \int_{0}^{T}\left(g, \tau_{h}\right)=0,
\end{aligned}
$$


for all $v_{h} \in H^{1}\left(V_{h}\right)$ such that $v_{h}(T)=0$, for all $\tau_{h} \in H^{1}\left(M_{h}\right)$ such that $\tau_{h}(T)=0$ and for all $q_{h} \in L^{2}\left(Q_{h}\right)$. A solution of the above equation satisfies $u_{h} \in H^{1}\left(V_{h}\right)$ and $\sigma_{h} \in H^{1}\left(M_{h}\right)$.

We then claim that problem (37) has a unique solution $\left(\tilde{u}_{h}, \tilde{\sigma}_{h}, \tilde{p}_{h}\right) \in X_{h}$. Indeed, when writing $\left(\tilde{u}_{h}, \tilde{p}_{h}, \tilde{\sigma}_{h}\right)$ with respect to a finite basis of $V_{h} \times Q_{h} \times M_{h}$, problem (37) can be expressed as a linear differential system. The degrees of freedom corresponding to the pressure can be eliminated. By a classical result of ODE, the resulting differential system has a unique solution, each components being in $H^{1}(0, T)$.

In order to prove (45), we choose $v_{h}=\tilde{u}_{h}(t), \tau_{h}=\tilde{\sigma}_{h}(t), q_{h}=\tilde{p}_{h}(t)$ in (37) and integrate from $t=0$ to $s$, with $0 \leq s \leq T$. We obtain

$$
\begin{aligned}
& \frac{\rho}{2}\left\|\tilde{u}_{h}(s)\right\|_{L^{2}(\Omega)}^{2}+\frac{\lambda}{4 \eta_{p}}\left\|\tilde{\sigma}_{h}(s)\right\|_{L^{2}(\Omega)}^{2}+2 \eta_{s} \int_{0}^{s}\left\|\epsilon\left(\tilde{u}_{h}\right)\right\|_{L^{2}(\Omega)}^{2} \\
& \quad+\sum_{K \in \mathcal{T}_{h}} \frac{\alpha h_{K}^{2}}{2 \eta_{p}} \int_{0}^{s}\left\|\nabla \tilde{p}_{h}\right\|_{L^{2}(K)}^{2}+\frac{1}{2 \eta_{p}} \int_{0}^{s}\left\|\tilde{\sigma}_{h}\right\|_{L^{2}(\Omega)}^{2} \\
& =\frac{\rho}{2}\left\|\tilde{u}_{h}(0)\right\|_{L^{2}(\Omega)}^{2}+\frac{\lambda}{4 \eta_{p}}\left\|\tilde{\sigma}_{h}(0)\right\|_{L^{2}(\Omega)}^{2}+\int_{0}^{s}\left(f, \tilde{u}_{h}\right)+\int_{0}^{s}\left(g, \tilde{\sigma}_{h}\right) .
\end{aligned}
$$

Using Young and Poincaré inequalities, there exists a constant $C$ such that

$$
\begin{aligned}
& \frac{\rho}{2}\left\|\tilde{u}_{h}(s)\right\|_{L^{2}(\Omega)}^{2}+\frac{\lambda}{4 \eta_{p}}\left\|\tilde{\sigma}_{h}(s)\right\|_{L^{2}(\Omega)}^{2}+\eta_{s} \int_{0}^{s}\left\|\epsilon\left(\tilde{u}_{h}\right)\right\|_{L^{2}(\Omega)}^{2} \\
& \quad+\sum_{K \in \mathcal{T}_{h}} \frac{\alpha h_{K}^{2}}{2 \eta_{p}} \int_{0}^{s}\left\|\nabla \tilde{p}_{h}\right\|_{L^{2}(K)}^{2}+\frac{\lambda}{2 \eta_{p}} \int_{0}^{s}\left\|\tilde{\sigma}_{h}\right\|_{L^{2}(\Omega)}^{2} \\
& \leq \frac{\rho}{2}\left\|\tilde{u}_{h}(0)\right\|_{L^{2}(\Omega)}^{2}+\frac{\lambda}{4 \eta_{p}}\left\|\tilde{\sigma}_{h}(0)\right\|_{L^{2}(\Omega)}^{2}+C\left(\int_{0}^{s}\|f\|_{L^{2}(\Omega)}^{2}+\int_{0}^{s}\|g\|_{L^{2}(\Omega)}^{2}\right) .
\end{aligned}
$$

It suffices to note that

$$
\begin{aligned}
& \left\|\tilde{u}_{h}(0)\right\|_{L^{2}(\Omega)}=\left\|i_{h} u_{0}\right\|_{L^{2}(\Omega)} \leq\left\|u_{0}\right\|_{L^{2}(\Omega)} \leq C\left\|u_{0}\right\|_{E_{1-1 / q, q}}, \\
& \left\|\tilde{\sigma}_{h}(0)\right\|_{L^{2}(\Omega)}=\left\|i_{h} \sigma_{0}\right\|_{L^{2}(\Omega)} \leq\left\|\sigma_{0}\right\|_{L^{2}(\Omega)} \leq C\left\|\sigma_{0}\right\|_{W^{1, r}},
\end{aligned}
$$

to obtain (45). 
We now prove the convergence result (46). Let

$$
\begin{array}{lll}
e_{u}=\tilde{u}-\tilde{u}_{h}=\Pi_{u}+C_{u}, & \Pi_{u}=\tilde{u}-i_{h} \tilde{u}, & C_{u}=i_{h} \tilde{u}-\tilde{u}_{h}, \\
e_{\sigma}=\tilde{\sigma}-\tilde{\sigma}_{h}=\Pi_{\sigma}+C_{\sigma}, & \Pi_{\sigma}=\tilde{\sigma}-i_{h} \tilde{\sigma}, & C_{\sigma}=i_{h} \tilde{\sigma}-\tilde{\sigma}_{h}, \\
e_{p}=\tilde{p}-\tilde{p}_{h}=\Pi_{p}+C_{p}, & \Pi_{p}=\tilde{p}-i_{h} \tilde{p}, & C_{p}=i_{h} \tilde{p}-\tilde{p}_{h},
\end{array}
$$

where $\left(\tilde{u}_{h}, \tilde{p}_{h}, \tilde{\sigma}_{h}\right)$ solve (37) and $(\tilde{u}, \tilde{p}, \tilde{\sigma})$ solve (39)-(44). Using the triangle inequality we have

$$
\left\|e_{u}, e_{\sigma}\right\|_{X_{h}} \leq\left\|\Pi_{u}, \Pi_{\sigma}\right\|_{X_{h}}+\left\|C_{u}, C_{\sigma}\right\|_{X_{h}} .
$$

Using classical interpolation results, we obtain

$$
\left\|\Pi_{u}, \Pi_{\sigma}\right\|_{X_{h}} \leq C h\|u, \sigma\|_{X}
$$

We now estimate $\left\|C_{u}, C_{\sigma}\right\|_{X_{h}}$. The solution of (39)-(44) satisfies

$$
\begin{aligned}
& \rho\left(\frac{\partial \tilde{u}}{\partial t}, v_{h}\right)+2 \eta_{s}\left(\epsilon(\tilde{u}), \epsilon\left(v_{h}\right)\right)-\left(\tilde{p}, \operatorname{div} v_{h}\right)+\left(\tilde{\sigma}, \epsilon\left(v_{h}\right)\right)-\left(f, v_{h}\right) \\
& +\left(\operatorname{div} \tilde{u}, q_{h}\right)+\frac{1}{2 \eta_{p}}\left(\tilde{\sigma}, \tau_{h}\right)+\frac{\lambda}{2 \eta_{p}}\left(\frac{\partial \tilde{\sigma}}{\partial t}, \tau_{h}\right)-\left(\epsilon(\tilde{u}), \tau_{h}\right)-\left(g, \tau_{h}\right)=0
\end{aligned}
$$

for all $\left(v_{h}, \tau_{h}, q_{h}\right) \in V_{h} \times M_{h} \times Q_{h}$. Subtracting (37) to the above equation, it follows that

$$
\begin{aligned}
& \rho\left(\frac{\partial e_{u}}{\partial t}, v_{h}\right)+2 \eta_{s}\left(\epsilon\left(e_{u}\right), \epsilon\left(v_{h}\right)\right)-\left(e_{p}, \operatorname{div} v_{h}\right)+\left(e_{\sigma}, \epsilon\left(v_{h}\right)\right) \\
& +\left(\operatorname{div} e_{u}, q_{h}\right)+\sum_{K \in \mathcal{T}_{h}} \frac{\alpha h_{K}^{2}}{2 \eta_{p}}\left(\nabla e_{p}-\nabla \tilde{p}, \nabla q_{h}\right)_{K} \\
& +\frac{1}{2 \eta_{p}}\left(e_{\sigma}, \tau_{h}\right)+\frac{\lambda}{2 \eta_{p}}\left(\frac{\partial e_{\sigma}}{\partial t}, \tau_{h}\right)-\left(\epsilon\left(e_{u}\right), \tau_{h}\right)=0,
\end{aligned}
$$


for all $\left(v_{h}, \tau_{h}, q_{h}\right) \in V_{h} \times M_{h} \times Q_{h}$. On the other hand, from the definition of $C_{u}$, $C_{\sigma}$ and $C_{p}$, we have

$$
\begin{aligned}
& \rho\left(\frac{\partial C_{u}}{\partial t}, C_{u}\right)+2 \eta_{s}\left(\epsilon\left(C_{u}\right), \epsilon\left(C_{u}\right)\right)-\left(C_{p}, \operatorname{div} C_{u}\right)+\left(C_{\sigma}, \epsilon\left(C_{u}\right)\right) \\
& \quad+\left(\operatorname{div} C_{u}, C_{p}\right)+\sum_{K \in \mathcal{T}_{h}} \frac{\alpha h_{K}^{2}}{2 \eta_{p}}\left(\nabla C_{p}, \nabla C_{p}\right)_{K} \\
& \quad+\frac{1}{2 \eta_{p}}\left(C_{\sigma}, C_{\sigma}\right)+\frac{\lambda}{2 \eta_{p}}\left(\frac{\partial C_{\sigma}}{\partial t}, C_{\sigma}\right)-\left(\epsilon\left(C_{u}\right), C_{\sigma}\right) \\
& =\rho\left(\frac{\partial\left(e_{u}-\Pi_{u}\right)}{\partial t}, C_{u}\right)+2 \eta_{s}\left(\epsilon\left(e_{u}-\Pi_{u}\right), \epsilon\left(C_{u}\right)\right)-\left(e_{p}-\Pi_{p}, \operatorname{div} C_{u}\right) \\
& \quad+\left(e_{\sigma}-\Pi_{\sigma}, \epsilon\left(C_{u}\right)\right)+\left(\operatorname{div}\left(e_{u}-\Pi_{u}\right), C_{p}\right)+\sum_{K \in \mathcal{T}_{h}} \frac{\alpha h_{K}^{2}}{2 \eta_{p}}\left(\nabla\left(e_{p}-\Pi_{p}\right), \nabla C_{p}\right)_{K} \\
& \quad+\frac{1}{2 \eta_{p}}\left(\left(e_{\sigma}-\Pi_{\sigma}\right), C_{\sigma}\right)+\frac{\lambda}{2 \eta_{p}}\left(\frac{\partial\left(e_{\sigma}-\Pi_{\sigma}\right)}{\partial t}, C_{\sigma}\right)-\left(\epsilon\left(e_{u}-\Pi_{u}\right), C_{\sigma}\right) .
\end{aligned}
$$

From the definition of $i_{h}$ (the $L^{2}$ projection onto the finite element spaces), we obviously have

$$
\left(\frac{\partial \Pi_{u}}{\partial t}, C_{u}\right)=0, \quad\left(\Pi_{\sigma}, C_{\sigma}\right)=0, \quad\left(\frac{\partial \Pi_{\sigma}}{\partial t}, C_{\sigma}\right)=0
$$

so that, using (74), (75) yields

$$
\begin{aligned}
\rho( & \left.\frac{\partial C_{u}}{\partial t}, C_{u}\right)+2 \eta_{s}\left(\epsilon\left(C_{u}\right), \epsilon\left(C_{u}\right)\right)+\sum_{K \in \mathcal{T}_{h}} \frac{\alpha h_{K}^{2}}{2 \eta_{p}}\left(\nabla C_{p}, \nabla C_{p}\right)_{K} \\
& +\frac{1}{2 \eta_{p}}\left(C_{\sigma}, C_{\sigma}\right)+\frac{\lambda}{2 \eta_{p}}\left(\frac{\partial C_{\sigma}}{\partial t}, C_{\sigma}\right) \\
= & 2 \eta_{s}\left(\epsilon\left(\Pi_{u}\right), \epsilon\left(C_{u}\right)\right)+\left(\Pi_{p}, \operatorname{div} C_{u}\right)-\left(\Pi_{\sigma}, \epsilon\left(C_{u}\right)\right) \\
& -\left(\operatorname{div}\left(\Pi_{u}\right), C_{p}\right)-\sum_{K \in \mathcal{T}_{h}} \frac{\alpha h_{K}^{2}}{2 \eta_{p}}\left(\nabla \Pi_{p}, \nabla C_{p}\right)_{K}+\left(\epsilon\left(\Pi_{u}\right), C_{\sigma}\right) \\
& +\sum_{K \in \mathcal{T}_{h}} \frac{\alpha h_{K}^{2}}{2 \eta_{p}}\left(\nabla \tilde{p}, \nabla C_{p}\right)_{K} \\
= & I_{1}+\cdots+I_{7} .
\end{aligned}
$$


It now remains to bound the terms $I_{1}, \ldots, I_{7}$ in the above equality. Using CauchySchwarz and Young's inequalities, we have

$$
\begin{aligned}
I_{1} & =2 \eta_{s}\left(\epsilon\left(\Pi_{u}\right), \epsilon\left(C_{u}\right)\right) \\
& \leq 2 \eta_{s}\left\|\epsilon\left(\Pi_{u}\right)\right\|_{L^{2}(\Omega)}\left\|\epsilon\left(C_{u}\right)\right\|_{L^{2}(\Omega)} \\
& \leq 3 \eta_{s}\left\|\epsilon\left(\Pi_{u}\right)\right\|_{L^{2}(\Omega)}^{2}+\frac{\eta_{s}}{3}\left\|\epsilon\left(C_{u}\right)\right\|_{L^{2}(\Omega)}^{2}
\end{aligned} .
$$

Similarly, we have

$$
\begin{aligned}
I_{2}=\left(\Pi_{p}, \operatorname{div} C_{u}\right) & \leq \frac{3}{4 \eta_{s}}\left\|\Pi_{p}\right\|_{L^{2}(\Omega)}^{2}+\frac{\eta_{s}}{3}\left\|\operatorname{div} C_{u}\right\|_{L^{2}(\Omega)}^{2} \\
& \leq \frac{3}{4 \eta_{s}}\left\|\Pi_{p}\right\|_{L^{2}(\Omega)}^{2}+\frac{\eta_{s}}{3}\left\|\epsilon\left(C_{u}\right)\right\|_{L^{2}(\Omega)}^{2},
\end{aligned}
$$

and

$$
I_{3}=-\left(\Pi_{\sigma}, \epsilon\left(C_{u}\right)\right) \leq \frac{3}{4 \eta_{s}}\left\|\Pi_{\sigma}\right\|_{L^{2}(\Omega)}^{2}+\frac{\eta_{s}}{2}\left\|\epsilon\left(C_{u}\right)\right\|_{L^{2}(\Omega)}^{2} .
$$

An integration by parts yields, since $\Pi_{u}=0$ on $\partial \Omega$

$$
\begin{aligned}
I_{4} & =\left(\operatorname{div}\left(\Pi_{u}\right), C_{p}\right)=-\left(\Pi_{u}, \nabla C_{p}\right)=-\sum_{K \in \mathcal{T}_{h}}\left(\Pi_{u}, \nabla C_{p}\right)_{K} \\
& \leq \sum_{K \in \mathcal{T}_{h}} \frac{\alpha h_{K}^{2}}{12 \eta_{p}}\left\|\nabla C_{p}\right\|_{L^{2}(K)}^{2}+\sum_{K \in \mathcal{T}_{h}} \frac{3 \eta_{p}}{\alpha h_{K}^{2}}\left\|\Pi_{u}\right\|_{L^{2}(K)}^{2} .
\end{aligned}
$$

Again, Cauchy-Schwarz and Young's inequalities yield

$$
\begin{aligned}
I_{5} & =-\sum_{K \in \mathcal{T}_{h}} \frac{\alpha h_{K}^{2}}{2 \eta_{p}}\left(\nabla \Pi_{p}, \nabla C_{p}\right)_{K} \\
& \leq \sum_{K \in \mathcal{T}_{h}} \frac{\alpha h_{K}^{2}}{12 \eta_{p}}\left\|\nabla C_{p}\right\|_{L^{2}(K)}^{2}+\frac{3 \alpha h^{2}}{4 \eta_{p}}\left\|\nabla \Pi_{p}\right\|_{L^{2}(\Omega)}^{2},
\end{aligned}
$$

and

$$
I_{6}=\left(\epsilon\left(\Pi_{u}\right), C_{\sigma}\right) \leq \eta_{p}\left\|\epsilon\left(\Pi_{u}\right)\right\|_{L^{2}(\Omega)}^{2}+\frac{1}{4 \eta_{p}}\left\|C_{\sigma}\right\|_{L^{2}(\Omega)}^{2} .
$$

Finally, we have

$$
\begin{aligned}
I_{8} & =\sum_{K \in \mathcal{T}_{h}} \frac{\alpha h_{K}^{2}}{2 \eta_{p}}\left(\nabla \tilde{p}, \nabla C_{p}\right)_{K} \\
& \leq \sum_{K \in \mathcal{T}_{h}} \frac{\alpha h_{K}^{2}}{12 \eta_{p}}\left\|\nabla C_{p}\right\|_{L^{2}(K)}^{2}+\frac{3 \alpha h^{2}}{4 \eta_{p}}\|\nabla \tilde{p}\|_{L^{2}(\Omega)}^{2} .
\end{aligned}
$$


The above estimates of $I_{1}, \ldots, I_{7}$ in (76) yield

$$
\begin{aligned}
& \rho\left(\frac{\partial C_{u}}{\partial t}, C_{u}\right)+\frac{1}{2} 2 \eta_{s}\left(\epsilon\left(C_{u}\right), \epsilon\left(C_{u}\right)\right)+\frac{1}{2} \sum_{K \in \mathcal{T}_{h}} \frac{\alpha h_{K}^{2}}{2 \eta_{p}}\left(\nabla C_{p}, \nabla C_{p}\right)_{K} \\
& \quad+\frac{1}{4 \eta_{p}}\left(C_{\sigma}, C_{\sigma}\right)+\frac{\lambda}{2 \eta_{p}}\left(\frac{\partial C_{\sigma}}{\partial t}, C_{\sigma}\right) \\
& \leq C\left(\left\|\epsilon\left(\Pi_{u}\right)\right\|_{L^{2}(\Omega)}^{2}+\left\|\Pi_{p}\right\|_{L^{2}(\Omega)}^{2}+\left\|\Pi_{\sigma}\right\|_{L^{2}(\Omega)}^{2}\right. \\
& \left.\quad+\sum_{K \in \mathcal{T}_{h}} \frac{1}{h_{K}^{2}}\left\|\Pi_{u}\right\|_{L^{2}(K)}^{2}+h^{2}\left\|\nabla \Pi_{p}\right\|_{L^{2}(\Omega)}^{2}+h^{2}\|\nabla \tilde{p}\|_{L^{2}(\Omega)}^{2}\right)
\end{aligned}
$$

where $C$ depends only on $\rho, \eta_{s}, \eta_{p}$ and $\alpha$. Time integration for $0 \leq s \leq T$ yields

$$
\begin{aligned}
& \frac{\rho}{2}\left\|C_{u}(s)\right\|_{L^{2}(\Omega)}^{2}+\eta_{s} \int_{0}^{s}\left\|\epsilon\left(C_{u}\right)\right\|_{L^{2}(\Omega)}^{2}+\frac{\lambda}{4 \eta_{p}}\left\|C_{\sigma}(s)\right\|_{L^{2}(\Omega)}^{2} \\
& \leq \frac{\rho}{2}\left\|C_{u}(0)\right\|_{L^{2}(\Omega)}^{2}+\frac{\lambda}{4 \eta_{p}}\left\|C_{\sigma}(0)\right\|_{L^{2}(\Omega)}^{2} \\
& \quad+C \int_{0}^{s}\left(\left\|\epsilon\left(\Pi_{u}\right)\right\|_{L^{2}(\Omega)}^{2}+\left\|\Pi_{p}\right\|_{L^{2}(\Omega)}^{2}+\left\|\Pi_{\sigma}\right\|_{L^{2}(\Omega)}^{2}\right. \\
& \quad+\sum_{K \in \mathcal{T}_{h}} \frac{1}{h_{K}^{2}}\left\|\Pi_{u}\right\|_{L^{2}(K)}^{2}+h^{2}\left\|\nabla \Pi_{p}\right\|_{L^{2}(\Omega)}^{2}+h^{2}\|\nabla \tilde{p}\|_{L^{2}(\Omega)}^{2}
\end{aligned}
$$

Using standard interpolation results, we finally obtain

$$
\left\|C_{u}, C_{\sigma}\right\|_{X_{h}}^{2} \leq C h^{2}\left(\|\tilde{u}, \tilde{\sigma}\|_{X}^{2}+\|\tilde{p}\|_{L^{q}\left(W^{1, r}\right)}^{2}+\left\|\nabla u_{0}\right\|_{L^{2}(\Omega)}^{2}+\left\|\nabla \sigma_{0}\right\|_{L^{2}(\Omega)}^{2}\right),
$$

where $C$ does not depend on $h, f, u_{0}, \sigma_{0}$ and $g$. Then, using continuous embeddings between interpolation spaces (see [37]), we have

$$
\left\|\nabla u_{0}\right\|_{L^{2}(\Omega)}^{2} \leq C\left\|u_{0}\right\|_{E_{1-1 / q, q}}^{2},
$$

where $C$ does not depend on $h, f, u_{0}, \sigma_{0}$ and $g$. Moreover, using the fact that the mapping

$$
\left(f, u_{0}, \sigma_{0}, g\right) \rightarrow(\tilde{u}, \tilde{\sigma}, \tilde{p})
$$

is continuous from

$$
Y \times L^{q}\left(W^{1, r}\right) \rightarrow L^{q}\left(W^{2, r}\right) \times L^{q}\left(W^{1, r}\right) \times L^{q}\left(W^{1, r}\right),
$$


we obtain

$$
\left\|C_{u}, C_{\sigma}\right\|_{X_{h}} \leq C h\left(\left\|f, u_{0}, \sigma_{0}\right\|_{Y}+\|g\|_{L^{q}\left(W^{1, r}\right)}\right),
$$

which concludes the proof.

\section{References}

1. Adams, R.A.: Sobolev Spaces. Academic, INC. London (1970)

2. Angenent, S.B.: Nonlinear analytic semiflows. Proc. R. Soc. Edinburgh Sect. A 115(1-2), 91107 (1990)

3. Baaijens, F.P.T.: Mixed finite element methods for viscoelastic flow analysis: a review. J. Non Newtonian Fluid Mech. 79, 361-385 (1998)

4. Baranger, J., Sandri, D.: Finite element approximation of viscoelastic fluid flow: existence of approximate solutions and error bounds. I. Discontinuous constraints. Numer. Math. 63(1), 13-27 (1992)

5. Baranger, J., Wardi, S.: Numerical analysis of a FEM for a transient viscoelastic flow. Comput. Methods Appl. Mech. Engrg. 125(1-4), 171-185 (1995)

6. Bird, R., Curtiss, C., Armstrong, R., Hassager, O.: Dynamics of polymeric liquids, vol. 1 and 2. Wiley, New York (1987)

7. Bonito, A.: Analysis and numerical simulation of viscoelastic flows : deterministic and stochastic models. PhD thesis, Département de Mathématiques, Ecole Polytechnique Fédérale de Lausanne (2006)

8. Bonito, A., Clément, Ph., Picasso, M.: Finite element analysis of a simplified stochastic Hookean dumbbells model arising from viscoelastic flows. M2AN Math. Model. Numer. Anal. 40(4), 785814 (2006)

9. Bonito, A., Clément, Ph., Picasso, M.: Mathematical analysis of a simplified Hookean dumbbells model arising from viscoelastic flows. J. Evol. Equ. 6(3), 381-398 (2006)

10. Bonito, A., Picasso, M., Laso, M.: Numerical simulation of $3 \mathrm{~d}$ viscoelastic flows with complex free surfaces. J. Comput. Phys. 215(2), 691-716 (2006)

11. Bonvin, J., Picasso, M., Stenberg, R.: GLS and EVSS methods for a three-field Stokes problem arising from viscoelastic flows. Comput. Methods Appl. Mech. Engrg. 190(29-30), 3893-3914 (2001)

12. Brezzi, F., Rappaz, J., Raviart, P.-A.: Finite-dimensional approximation of nonlinear problems. I. Branches of nonsingular solutions. Numer. Math. 36(1), 1-25 (1980/81)

13. Brezzi, F., Rappaz, J., Raviart, P.-A.: Finite-dimensional approximation of nonlinear problems. II. Limit points. Numer. Math. 37(1), 1-28 (1981)

14. Buffoni, B., Toland, J.: Analytic theory of global bifurcation. Princeton Series in Applied Mathematics. Princeton University Press, Princeton (2003) An introduction

15. Caloz, G., Rappaz J.: Numerical analysis for nonlinear and bifurcation problems. In: Handbook of numerical analysis, vol. V, Handb. Numer. Anal., V, pp. 487-637. North-Holland, Amsterdam (1997)

16. Cartan, H.: Calcul différentiel. Hermann, Paris (1967)

17. Ciarlet, P.G., Lions, J.-L.: (eds.) Handbook of numerical analysis. Vol. II. Handbook of Numerical Analysis, II. North-Holland, Amsterdam (1991) Finite element methods. Part 1

18. Da Prato, G., Sinestrari, E.: Differential operators with nondense domain. Ann. Scuola Norm. Sup. Pisa Cl. Sci. (4), 14(2), 285-344 (1988), 1987

19. Di Blasio, G.: Linear parabolic evolution equations in $L^{p}$-spaces. Ann. Mat. Pura Appl. 138(4), 55104 (1984)

20. Dore, G.: $L^{p}$ regularity for abstract differential equations. In: Functional analysis and related topics, 1991 (Kyoto), vol. 1540 of Lecture Notes in Math., pp. 25-38. Springer, Berlin (1993)

21. Dore, G., Venni, A.: On the closedness of the sum of two closed operators. Math. Z. 196(2), 189201 (1987)

22. Ervin, V.J., Heuer, N.: Approximation of time-dependent, viscoelastic fluid flow: Crank-Nicolson, finite element approximation. Numer. Methods Partial Diff. Eqn. 20(2), 248-283 (2004)

23. Ervin, V.J., Miles, W.W.: Approximation of time-dependent viscoelastic fluid flow: SUPG approximation. SIAM J. Numer. Anal. 41(2), 457-486 (electronic) (2003)

24. Farhloul, M., Zine, A.M.: A new mixed finite element method for viscoelastic fluid flows. Int. J. Pure Appl. Math. 7(1), 93-115 (2003)

25. Fernández-Cara, E., Guillén, F., Ortega, R.R.: Mathematical modeling and analysis of viscoelastic fluids of the Oldroyd kind. In: Handbook of numerical analysis, vol. VIII, Handb. Numer. Anal., VIII, pp. 543-661. North-Holland, Amsterdam (2002) 
26. Fortin, A., Guénette, R., Pierre, R.: On the discrete EVSS method. Comput. Methods Appl. Mech. Engrg. 189(1), 121-139 (2000)

27. Fortin, M., Pierre, R.: On the convergence of the mixed method of Crochet and Marchal for viscoelastic flows. Comput. Methods Appl. Mech. Engrg. 73(3), 341-350 (1989)

28. Fujiwara, D., Morimoto, H.: An $L_{r}$-theorem of the Helmholtz decomposition of vector fields. J. Fac. Sci. Univ. Tokyo Sect. IA Math. 24(3), 685-700 (1977)

29. Galdi, G.P.: An introduction to the mathematical theory of the Navier-Stokes equations. vol. I, volume 38 of Springer Tracts in Natural Philosophy. Springer-Verlag, New York, (1994) Linearized steady problems

30. Galdi, G.P., Simader, C.G., Sohr, H.: A class of solutions to stationary Stokes and Navier-Stokes equations with boundary data in $W^{-1 / q, q}$. Math. Ann. 331(1), 41-74 (2005)

31. Giesekus, H.: A simple constitutive equation for polymer fluids based on the concept of deformationdependent tensorial mobility. J. Non Newtonian Fluid Mech. 11(1-2), 69-109 (1982)

32. Giga, Y.: Analyticity of the semigroup generated by the Stokes operator in $L_{r}$ spaces. Math. Z. 178(3), 297-329 (1981)

33. Glowinski, R.: Finite element methods for incompressible viscous flow. In Handbook of numerical analysis, Vol. IX, Handb. Numer. Anal., IX, pp. 3-1176. North-Holland, Amsterdam (2003)

34. Guillopé, C., Saut, J.-C.: Existence results for the flow of viscoelastic fluids with a differential constitutive law. Nonlinear Anal. 15(9), 849-869 (1990)

35. Keunings, R.: Micro-marco methods for the multi-scale simulation of viscoelastic flow using molecular models of kinetic theory. Rheol. Rev., pp. 67-98 (2004)

36. Lions, P.L., Masmoudi, N.: Global solutions for some Oldroyd models of non-Newtonian flows. Chinese Ann. Math. Ser. B 21(2), 131-146 (2000)

37. Lunardi, A.: Analytic semigroups and optimal regularity in parabolic problems. Progress in Nonlinear Differential Equations and their Applications. 16. Birkhäuser Verlag, Basel (1995)

38. Machmoum, A., Esselaoui, D.: Finite element approximation of viscoelastic fluid flow using characteristics method. Comput. Methods Appl. Mech. Engrg. 190(42), 5603-5618 (2001)

39. Najib, K., Sandri, D.: On a decoupled algorithm for solving a finite element problem for the approximation of viscoelastic fluid flow. Numer. Math. 72(2), 223-238 (1995)

40. Öttinger, H.C.: Stochastic processes in polymeric fluids. Springer, Berlin (1996)

41. Owens, R.G., Phillips, T.N.: Computational rheology. Imperial College Press, London (2002)

42. Phan-Thien, N., Tanner, R.I.: A new constitutive equation derived from network theory. J. Non Newtonian Fluid Mech. 2(4), 353-365 (1977)

43. Picasso, M., Rappaz, J.: Existence, a priori and a posteriori error estimates for a nonlinear three-field problem arising from Oldroyd-B viscoelastic flows. M2AN Math. Model. Numer. Anal. 35(5), 879897 (2001)

44. Prüss, J.: Evolutionary integral equations and applications, volume 87 of Monographs in Mathematics. Birkhäuser Verlag, Basel (1993)

45. Renardy, M.: Existence of slow steady flows of viscoelastic fluids of integral type. Z. Angew. Math. Mech. 68(4), T40-T44 (1988)

46. Sandri, D.: Analyse d'une formulation à trois champs du problème de Stokes. RAIRO Modél. Math. Anal. Numér. 27(7), 817-841 (1993)

47. Sandri, D.: Finite element approximation of viscoelastic fluid flow: existence of approximate solutions and error bounds. Continuous approximation of the stress. SIAM J. Numer. Anal. 31(2), 362377 (1994)

48. Simader, C.G., Sohr, H.: A new approach to the Helmholtz decomposition and the Neumann problem in $L^{q}$-spaces for bounded and exterior domains. In: Mathematical problems relating to the NavierStokes equation. vol. 11 of Ser. Adv. Math. Appl. Sci. pp. 1-35. World Scientific Publishing, River Edge (1992)

49. Sinestrari, E.: On the abstract Cauchy problem of parabolic type in spaces of continuous functions. J. Math. Anal. Appl. 107(1), 16-66 (1985)

50. Sobolevskiǔ, P.E.: Coerciveness inequalities for abstract parabolic equations. Dokl. Akad. Nauk SSSR 157, 52-55 (1964)

51. Sobolevskil̆, P.E.: Fractional powers of coercively positive sums of operators. Dokl. Akad. Nauk SSSR 225(6), 1271-1274 (1975)

52. Solonnikov, V.A.: Estimates of the Solutions of a Nonstationary Linearized System of Navier-Stokes Equations, pp. 214-317. American Mathematical Society, Rhode island (1968)

53. Yosida, K.: Functional analysis, volume 123 of Grundlehren der Mathematischen Wissenschaften (Fundamental Principles of Mathematical Sciences). Springer, Berlin (1980) 